\title{
Individualised stepwise adaptive treatment for 3-6-year-old preschool children impaired by attention-deficit/hyperactivity disorder (ESCApreschool): study protocol of an adaptive intervention study including two randomised controlled trials within the consortium ESCAlife
}

Katja Becker ${ }^{1,2^{*}}$, Tobias Banaschewski ${ }^{3}$, Daniel Brandeis ${ }^{3}$, Christina Dose ${ }^{4,5}$, Christopher Hautmann ${ }^{4,5}$, Martin Holtmann ${ }^{6}$, Thomas Jans ${ }^{7}$, Lea Jendreizik ${ }^{4}$, Carolin Jenkner ${ }^{8}$, Katja John ${ }^{1}$, Johanna Ketter ${ }^{1}$, Sabina Millenet ${ }^{3}$, Ursula Pauli-Pott ${ }^{1,2}$, Tobias Renner ${ }^{9}$, Marcel Romanos ${ }^{7}$, Anne-Katrin Treier $^{3}$, Elena von Wirth ${ }^{4,5}$,

Anne-Kathrin Wermter ${ }^{1,2}$ and Manfred Döpfner ${ }^{4,5}$

\begin{abstract}
Background: Attention-deficit/hyperactivity disorder (ADHD) is a psychosocially impairing and cost-intensive mental disorder, with first symptoms occurring in early childhood. It can usually be diagnosed reliably at preschool age. Early detection of children with ADHD symptoms and an early, age-appropriate treatment are needed in order to reduce symptoms, prevent secondary problems and enable a better school start. Despite existing ADHD treatment research and guideline recommendations for the treatment of ADHD in preschool children, there is still a need to optimise individualised treatment strategies in order to improve outcomes. Therefore, the ESCApreschool study (Evidence-Based, Stepped Care of ADHD in Preschool Children aged 3 years and 0 months to 6 years and 11 months of age (3;0 to 6;11 years) addresses the treatment of 3-6-year-old preschool children with elevated ADHD symptoms within a large multicentre trial. The study aims to investigate the efficacy of an individualised stepwiseintensifying treatment programme.

Methods: The target sample size of ESCApreschool is 200 children (boys and girls) aged 3;0 to 6;11 years with an ADHD diagnosis according to Diagnostic and Statistical Manual of Mental Disorders, Fifth Edition (DSM-5) or a diagnosis of oppositional defiant disorder (ODD) plus additional substantial ADHD symptoms. The first step of the adaptive, stepped care design used in ESCApreschool consists of a telephone-assisted self-help (TASH) intervention for parents. Participants are randomised to either the TASH group or a waiting control group. The treatment in step (Continued on next page)
\end{abstract}

\footnotetext{
* Correspondence: katja.becker@uni-marburg.de

1 Department of Child and Adolescent Psychiatry, Psychosomatics and

Psychotherapy, Medical Faculty of the Philipps-University Marburg,

Hans-Sachs-Str. 6, 35039 Marburg, Germany

${ }^{2}$ Center for Mind, Brain and Behavior (CMBB), University of Marburg and

Justus Liebig University Giessen, Marburg, Germany

Full list of author information is available at the end of the article
}

(c) The Author(s). 2020 Open Access This article is distributed under the terms of the Creative Commons Attribution 4.0 International License (http://creativecommons.org/licenses/by/4.0/), which permits unrestricted use, distribution, and reproduction in any medium, provided you give appropriate credit to the original author(s) and the source, provide a link to the Creative Commons license, and indicate if changes were made. The Creative Commons Public Domain Dedication waiver (http://creativecommons.org/publicdomain/zero/1.0/) applies to the data made available in this article, unless otherwise stated. 
(Continued from previous page)

2 depends on the outcome of step 1: TASH responders without significant residual ADHD/ODD symptoms receive booster sessions of TASH. Partial or non-responders of step 1 are randomised again to either parent management and preschool teacher training or treatment as usual.

Discussion: The ESCApreschool trial aims to improve knowledge about individualised treatment strategies for preschool children with ADHD following an adaptive stepped care approach, and to provide a scientific basis for individualised medicine for preschool children with ADHD in routine clinical care.

Trial registration: The trial was registered at the German Clinical Trials Register (DRKS) as a Current Controlled Trial under DRKS00008971 on 1 October 2015. This manuscript is based on protocol version 3 (14 October 2016).

Keywords: Attention-deficit/hyperactivity disorder, ADHD, Preschool children, Stepped care, Adaptive treatment, Telephone-assisted self-help, Behaviour therapy, Kindergarten, ESCAlife

\section{Background}

Attention-deficit/hyperactivity disorder (ADHD) is a highly prevalent, early-onset, persistent neurodevelopmental disorder, which is associated with psychosocial functional impairment and a markedly reduced subjective health-related quality of life [1-3]. According to Diagnostic and Statistical Manual of Mental Disorders, Fifth Edition (DSM-5) or International Classification of Diseases, Tenth Edition (ICD-10) criteria, it is characterised by ageinappropriate, pervasive and persistent inattentiveness, impulsivity and/or motor restlessness [4, 5]. ADHD symptoms can be observed as early as the preschool years, with an estimated prevalence of $1.5-6 \%$ among preschool children $[6,7]$. For the diagnosis of ADHD, clinically relevant functional impairment must be present in different settings, e.g. in the family and at preschool. In addition, comorbidity in preschool children with ADHD is common, with oppositional defiant disorder (ODD), communication disorder and anxiety disorders being the most prevalent comorbid conditions [8]. Early interventions have been shown to be particularly helpful and might prevent the development of secondary symptoms as well as school failure [9-11]. International and national treatment guidelines [12-14] recommend a combination of multiple, individually adapted treatment components (i.e. multimodal therapy). However, compared with school-age children, treatment with immediate-release methylphenidate has been shown to be less effective in preschool children (i.e. effect sizes were considerably smaller), to cause more adverse events and to be less accepted by parents [15]. In contrast, psychosocial treatment may be most powerful at this early age, as it can positively influence parental scaffolding during early self-regulation development and prevent the development of coercive cycles of negative parent-child interactions. Accordingly, clinical guidelines recommend psychosocial interventions in the family and the preschool as the treatment of choice for preschool children with ADHD [12-14].

Parent counselling and parent management training have been found to be effective treatments for children of this age group [12, 16, 17]. Recent metaanalyses on the efficacy and effectiveness of psychosocial interventions in preschool children with disruptive behaviour disorders (DBD), including ADHD, showed medium to large effects on child behaviour outcomes. Based on 13 studies, Charach et al. [18] found a moderate overall effect (standardised mean difference $[S M D]=0.75$ ) of parenting training on parent-reported DBD symptoms; the effect size for core symptoms of ADHD was SMD = 0.77 (five included studies). Similarly, a meta-analysis of 36 randomised control trials (RCTs) on a broader range of psychosocial interventions resulted in a large overall effect on parent-, teacher- and observer-reported DBD symptoms (Hedges' $g=0.82$ ) and a medium effect $(g=0.61)$ on hyperactivity/impulsivity symptoms in particular [19]. The analysis included behavioural and non-behavioural treatments, with the former showing significantly larger effects [19]. Even though clinical guidelines recommend preschool interventions, so far, preschool teacher training as well as preschool-based interventions are rare. The Cologne group led by Döpfner reported that their indicated prevention programme addressing preschool teachers was effective $[9,20$, 21]. The measured effects were largely maintained at 1-year follow-up (e.g. [9, 22]).

However, previous studies in preschool children with ADHD are subject to some limitations. For instance, the evidence regarding the value of these interventions is limited to unblinded ratings made by individuals who are likely to be invested in the treatment success. Evidence of efficacy from wellcontrolled trials using blinded assessments of outcomes is still lacking [23]. Furthermore, the validity of the available RCTs is limited by the design characteristics, as most of the RCTs used no treatment as a control condition, rather than treatment as usual (TAU) or non-specific support. Therefore, some of these results cannot be generalised [18, 19]. 
Another critical issue in the treatment of preschool children with ADHD is that unfortunately, not all parents are willing or able to procure treatment for their children. Frequent reasons why parents do not start or complete interventions include a lack of problem awareness, a lack of availability of psychotherapy, or other problems such as transport, childcare, work commitments, financial burden, or stigma (e.g. [24, 25]). Besides and despite these treatment barriers, the need for intervention still exceeds the number of available treatment options, and treatment resources are sparse [24]. Therefore, it is important to focus on therapies and dissemination methods that help to overcome these barriers. There is evidence that self-directed, bibliographic interventions and telephone- or web-based assistance might be one way forward [18, 26, 27]. Self-directed interventions have been shown to be effective in reducing parent-rated externalising behaviour problems [28, 29]. Some studies indicate that the effects of such interventions may be improved by minimal therapeutic support (e.g. by telephone; see [28]). For example, Kierfeld et al. [30] successfully demonstrated the effects of a telephone-assisted self-help (TASH) intervention for parents of preschool children with ADHD and other externalising behaviour problems. The effects were maintained at 1-year follow-up [31]. An effectiveness study on a TASH intervention for parents of 6-12-yearold children with ADHD found a significant reduction of ADHD and comorbid symptoms under routine care conditions [32]. Moreover, TASH for parents was found to enhance effects of methylphenidate treatment in a sample of children with ADHD [33]. Interestingly, behavioural and non-behavioural TASH interventions seem to have similar effects [34].

An open research question is which of the different treatment components (e.g. behaviour therapy, selfdirected interventions) should be offered following obligatory psychoeducation, and in which order. In this respect, a stepped care approach in which treatment is individually adapted according to symptom strength, comorbid symptoms, specific family needs and treatment response is suggested [12]. However, empirical evidence for the efficacy of adaptive treatment strategies for patients with an ADHD diagnosis in general, and in preschool children in particular, is sparse. A study assessing an adaptive multimodal treatment in school-age children found that both behaviour therapy and a combination of behaviour therapy and pharmacotherapy are effective in the treatment of ADHD [35], with effects persisting at an 18-month follow-up [36]. Whereas the efficacy of different singular interventions is also well documented in preschool children, a stepwise approach with individualised adaptive treatment strategies has not been empirically validated for this age group. Therefore, a stepped care approach for 3-6-year-old preschool children is being evaluated within the trial ESCApreschool (EvidenceBased, Stepped Care of ADHD in Preschool Children aged 3 years and 0 months to 6 years and 11 months of age (3;0 to $6 ; 11$ years). The results aim to improve the knowledge about individualised treatment strategies for preschool children with ADHD. The evaluation of a stepwise approach in routine care is of particular importance for clinical practice.

\section{Methods and Design}

ESCApreschool is part of a multicentre consortium studying stepped care approaches for the treatment of ADHD along the lifespan (ESCAlife: Evidence-Based Stepped Care of ADHD along the Lifespan, coordinator Tobias Banaschewski). ESCAlife encompasses stepped care designs in different age groups (preschool age, school age, adolescents, adults), each focusing on the different specific needs in the respective life phases, including 6-12-year-old school children (ESCAschool [37]), 12-17-year-old adolescents (ESCAadol [38]) and 16-45year-old adults (ESCAlate [39]). With regard to design and methodology, the single studies overlap to allow for the examination of selected research questions across all age groups.

\section{Objectives, study design and trial flow}

ESCApreschool aims to examine the efficacy of an individualised stepwise-intensifying treatment approach based on evidence-based behavioural interventions in patients with ADHD or patients with ODD and additional ADHD symptoms, aged 3;0 to 6;11 years, who attend preschool. Different treatment strategies are investigated for children who respond to a low-threshold TASH intervention and those who do not. A further question is to determine precisely which families benefit from the low-threshold TASH intervention or more intensive behaviour therapy and which families do not, and to identify the predictors and moderators of treatment response. Therefore, the secondary objective is to examine the predictability of treatment response by psychological and biological variables.

The multicentre study is designed as a stepwise (two steps) adaptive treatment study including two RCTs. In the adaptive design, the second step of the trial (step 2) depends on the outcome of step 1.

Step 1 of the ESCApreschool study consists of a randomised waitlist-controlled trial which provides the parents (and optionally also the preschool teachers) of the participating children (planned number of $N=200$ children) with a 3-month TASH intervention. The parents (and preschool teachers) are randomised to receive this treatment either immediately at the beginning of the trial or after a 3-month waiting period. 
The intervention provided in step 2 of the trial depends on the outcome of the low-threshold TASH intervention. If children fully respond to this intervention, their parents (and preschool teachers) receive TASH booster sessions in step 2 . If children do not or only partially respond to TASH, that is, if they show persisting ADHD and/or ODD symptoms, they are randomised to receive either parent management and preschool teacher training (PMPTT) or TAU. For an overview of the trial flow, please refer to Fig. 1.

The sample sizes and response rates displayed in the figure are estimations and therefore differ from the actual recruitment and response rates.

Step 1 lasts for 3-6 months depending on the allocation of the participants ( 3 months in the TASH group; 6 months in the waiting control group, which undergoes a 3 -month waiting period followed by the 3-month TASH intervention period). Step 2 lasts for 6 months, and the follow-up period lasts for 3 months.

Measurements are taken at $\mathrm{T} 0$ and $\mathrm{T} 1(\mathrm{~T} 0=$ screening of inclusion and exclusion criteria and assessment of ADHD and ODD criteria; T1 = baseline assessment as described below). Further measurements ensue after step 1 (T2), after the second treatment phase in step 2 (T3), and 3 months after the end of the treatment (follow-up examination; T4). Families who are randomised to the waiting control group take part in an additional assessment after the waiting period (T2b1). Participants who discontinue the intervention during one of the treatment phases are also invited for the follow-up assessment (T4) in order to monitor their development. Additionally, data are collected during the therapeutic process.

Clinical assessments of ADHD and ODD symptom severity are completed by trained experienced clinicians (further referred to as "blinded clinicians"), who are blind to the patients' assignment to treatment condition but not to the assessment time point (T1-T4). The inclusion of participants into the study, as well as their classification as full responders or partial/non- responders to step 1, are based on these clinical interviews. For validation purposes, all interviews are recorded and a subsample of the recordings is subsequently rated by a clinician who is blind to both the treatment condition and the assessment time point.

\section{Trial sites}

At the start of the study, a total of six trial centres located at departments of child and adolescent psychiatry at university hospitals in Germany (Cologne, Hamm, Mannheim, Marburg, Tübingen, Würzburg) contributed to this multicentre trial.

The leading and coordinating centre of the ESCApreschool study is Marburg (Principal Investigator [PI] Katja Becker). Each of the six centres was expected to enrol between 30 and 40 patients in order to achieve a total sample size of $N=200$ patients. To compensate for low recruiting numbers, three additional trial centres were included in 2018 (Aachen [Kerstin Konrad], Göttingen [Luise Poustka], Neuruppin [Michael Kölch]).

The TASH work group at the Cologne University Hospital (Manfred Döpfner, also Co-PI of ESCApreschool) is responsible for delivering TASH (which is provided centrally from Cologne for all participants). All other diagnostic procedures and treatments are provided at the respective study centres. On-site therapists who have been trained and who are supervised by the Cologne work group perform the behaviour therapy. Responsibility for data management, archiving and monitoring, as well as biometrics and project management, lies with the Centre for Clinical Studies in Freiburg.

\section{Participants}

A total of 200 girls and boys, aged 3;0 to 6;11 years, with either a diagnosis of ADHD or substantial ADHD symptoms combined with a diagnosis of ODD are the study's target group. Inclusion and exclusion criteria are shown in Table 1 (see Measures section for a more detailed description of the instruments). These data are assessed at

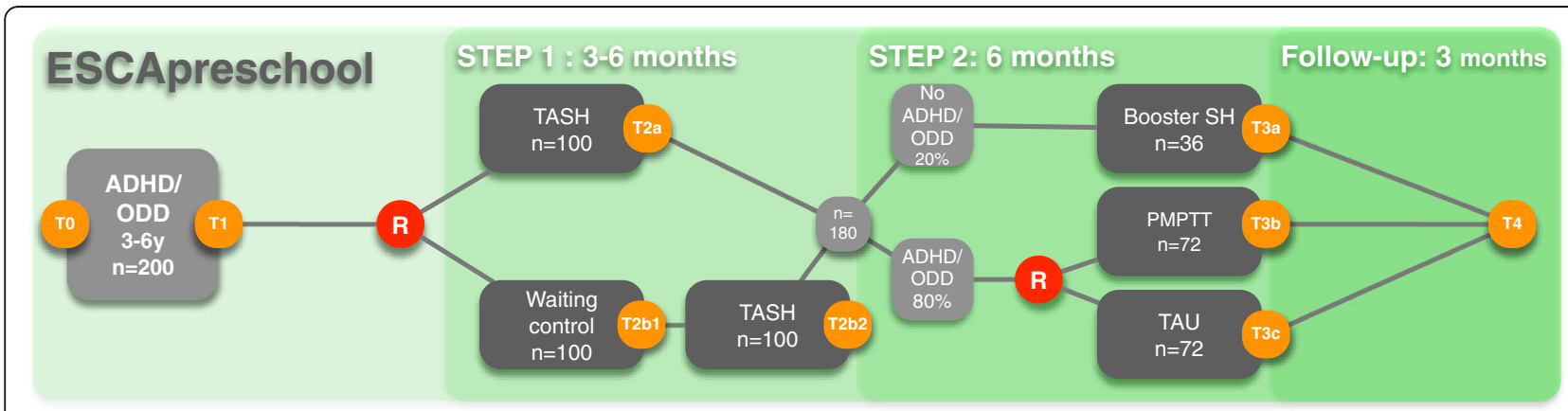

Fig. 1 Flow chart. ADHD = Attention-Deficit/Hyperactivity Disorder; ODD = Oppositional Defiant Disorder, T0 to T4=Assessment Time Points; R= Randomisation; TASH = Telephone-Assisted Self-Help for Parents and Preschool Teachers; PMPTT = Parent Management and Preschool Teacher Training, TAU = Treatment as Usual, Booster $\mathrm{SH}=$ Booster Self-Help 
Table 1 Inclusion and exclusion criteria

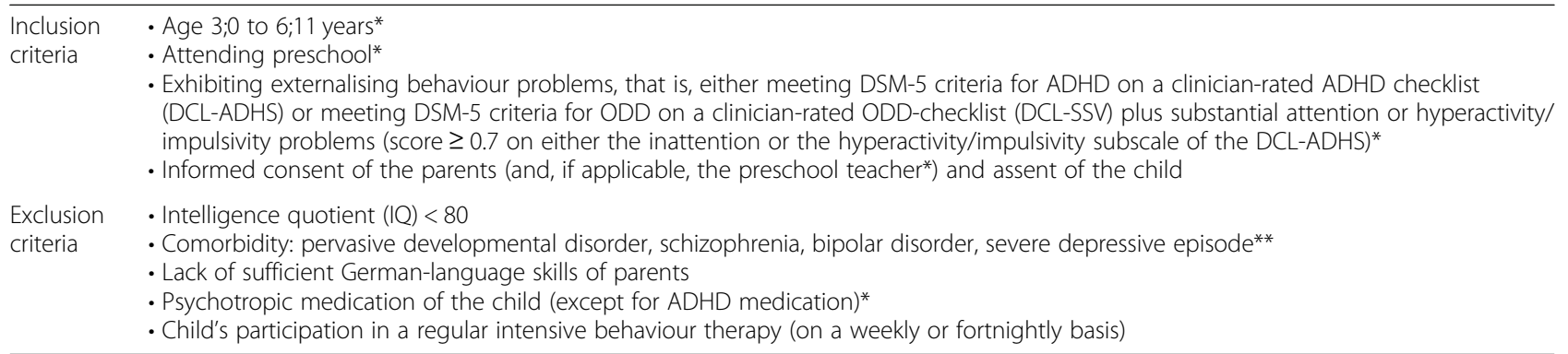

baseline through an interview gathering baseline characteristics as well as sociodemographic data. Patients are recruited through the centres' outpatient clinics, which are highly experienced in the treatment of ADHD or preschool children. Further recruitment strategies include the dissemination of information regarding the study at local conferences, and by contacting paediatricians, child and adolescent psychiatrists, child and adolescent psychologists and child guidance centres. Additionally, information is provided to other counselling centres, ADHD self-help groups and preschool teachers either in writing, in person, or through lectures. In Germany, the local health authorities organise a mandatory health examination for preschool children shortly before school entry. Therefore, the respective local health authorities are also informed about the study. All of these recruitment activities are flanked by homepage information, local public lectures and repeated advertisement actions providing information about the study (postings, flyers, bus advertisements, newspaper articles) in order to reach parents directly.

"Some changes have been made since the first version of the study protocol (9 June 2015). First, we initially planned an age span of $3 ; 0$ to $5 ; 11$ years. However, this resulted in the exclusion of 6 -year-olds who were still attending preschool within the overall ESCAlife study. Thus, this inclusion criterion was changed to $3 ; 0$ to $6 ; 11$ years (Note to File G005 22 September 2016, positive Ethics Committee vote 08 November 2016). Second, the first version of the study protocol comprised an additional inclusion criterion of a time span from at least 9 months before enrolment in primary school. Due to recruitment problems, this criterion was changed to "attending preschool” (Note to File G003 25 May 2016; positive Ethics Committee vote 3 June 2016). Third, an inclusion criterion in the first version of the trial protocol was the presence of an ADHD diagnosis according to DSM-5, assessed with a clinician-rated ADHD checklist (DCL-ADHS). However, after study start, we realised that at preschool age, the differentiation between a diagnosis of ADHD and a diagnosis of ODD plus additional ADHD symptoms might be blurred. Therefore, this inclusion criterion was changed to that mentioned in the table (Note to file G002, 25 May 2016, positive Ethics Committee vote 3 June 2016).

Fourth, the initial inclusion criterion "informed consent of preschool teacher" was withdrawn due to the fact that in some centres, it was not permitted to contact preschool teachers directly. Therefore, in the present version of the study protocol, while it is preferable to include a preschool teacher in the study (if parents agree to their being contacted), this is not a prerequisite or an inclusion criterion (Note to File G003 25 May 2016; positive Ethics Committee vote 3 June 2016). Fifth, initially, an exclusion criterion was "current medication for ADHD or other psychotropic medication”. After realising that this exclusion criterion leads to an exclusion of severe cases with a current ADHD medication, this criterion was changed to "psychotropic medication of the child (except for ADHD medication)" (Note to File G003 25 May 2016, positive Ethics Committee vote 3 June 2016).

This table gives the inclusion and exclusion criteria of Version 3 of the Trial Protocol (V03, 14. October 2016) including all amendments G001-G005.

** The comorbid conditions which are defined as exclusion criteria are the same as those for the different trials within the ESCAlife consortium, including some diagnoses which are unlikely to appear at preschool age.

Patients are included if they meet the eligibility criteria (see Table 1). Parents (and, if applicable, preschool teachers) must give their informed consent and children their assent for study participation.

\section{Response criteria}

The response criteria correspond to the child's symptom inclusion criteria (see Table 1). Partial or nonresponders have ADHD (DSM-5) assessed with the clinician-rated ADHD Checklist (DCL-ADHS) or ODD (DSM-5) assessed with the clinician-rated ODD Checklist (DCL-SSV) plus substantial ADHD symptoms (defined by a score of $\geq 0.7$ on either of the two subscales [hyperactivity/impulsivity or inattention] of the DCLADHS). Full responders fulfil neither of these conditions and no longer have ADHD or ODD with substantial ADHD symptoms. 


\section{Data handling}

All legal requirements pertaining to the protection of personal data have been met. Upon enrolment, every participating child is assigned with a study-specific identification code. To ensure complete pseudonymisation, all study data from patients and their parents are stored under their assigned code. This is not shared, with the sole exception of transmission of contact details to members of the Cologne group providing the TASH intervention, following consent from participating parents. Only the PI and the study coordinators at each site have access to the patient identification list. The Clinical Trials Unit (CTU) Freiburg provides an electronic remote data entry system (RDE-LIGHT), in which information is entered by specifically trained personnel under the study code. To prevent unauthorised access to confidential participant information, built-in security features encrypt all data before transmission to and from the CTU. Users who enter data into the system are registered with the CTU and receive an individual ID and password to gain access to the system, in order to prevent unauthorised access to patient data. Data processing at the CTU is limited to authorised personnel who are familiar with the data handling procedures according to the study protocol.

\section{Interventions}

\section{Telephone-assisted self-help (TASH)}

In step 1, all participants receive a 3-month behaviour therapy-oriented TASH intervention for parents of children with externalising behaviour problems. Additionally, if both the parents and the preschool teacher agree, preschool teachers also receive the intervention [40, 41]. The intervention is based on the Therapy Programme for Children with Hyperactive and Oppositional Problem Behaviour (Therapieprogramm für Kinder mit hyperkinetischem und oppositionellem Problemverhalten - THOP
[42]) and the German self-help book Wackelpeter $\mathcal{E}$ Trotzkopf [43]. Both the parent and the preschool teacher programme consist of self-help booklets on externalising behaviour problems and behaviour modification techniques, which are sent to the participants through the post. Additionally, they receive telephone consultations with a therapist in advanced training for child and adolescent psychotherapy, who is supervised by senior supervisors. These consultations serve to support the parents and preschool teachers with the implementation of the interventions into their daily routines $[40,41]$.

Parent TASH programmes similar to that used in the present study have already been shown to reduce behaviour problems in preschool- and school-age children (e.g. [30, 32-34]). For the current study, the parent booklets were revised and adapted to address the specific needs of families of preschool children. The parents receive eight booklets and ten telephone consultations, each lasting for approximately 30 minutes. The TASH programme for the preschool teachers consists of four newly developed booklets and four telephone consultations of up to 60 minutes each. The appointments for the telephone consultations are set on an individual basis. The contents of the booklets are described in Table 2 and Additional file 1: Table S1.

For the preschool teachers, the interventions originally developed for the family environment have been adapted to the preschool environment. Moreover, the booklets for the preschool teachers cover information on improving environmental conditions, which might help the child to deal with his or her behaviour problems, and on constructive cooperation with parents (see Additional file 1: Table S1).

If children are full responders to the TASH intervention, two additional telephone consultations for the parents and, optionally, one additional telephone

Table 2 Overview of the telephone-assisted self-help (TASH) booklets for parents of preschool children

\begin{tabular}{|c|c|c|}
\hline & Title & Content \\
\hline 1 & Taking a close look at our problems & $\begin{array}{l}\text { Defining individual problem behaviour and psychoeducation regarding coercive parent-child } \\
\text { interactions }\end{array}$ \\
\hline 2 & What is ADHD? & $\begin{array}{l}\text { Psychoeducation on ADHD symptoms in preschoolers, associated problems, reasons for ADHD, } \\
\text { the developmental course of ADHD, and treatment alternatives }\end{array}$ \\
\hline 3 & Learning to like each other again & $\begin{array}{l}\text { Encouragement of positive parent-child interactions by focusing on positive traits and positive ex } \\
\text { periences with the child and by actively creating more positive interactions and experiences with } \\
\text { the child }\end{array}$ \\
\hline 4 & $\begin{array}{l}\text { Clear daily structures - gathering energy and } \\
\text { implementing clear rules }\end{array}$ & $\begin{array}{l}\text { Implementation of well-structured daily and weekly routines, strategies of parental stress manage } \\
\text { ment, and reflecting on and implementing family rules }\end{array}$ \\
\hline 5 & $\begin{array}{l}\text { Make effective requests and do not skimp on } \\
\text { praise }\end{array}$ & Making effective requests, praise and positive consequences for following rules \\
\hline 6 & The need for consequences & Appropriate negative consequences for breaking rules \\
\hline 7 & If praise is not enough: reward systems & Implementation of reward symptoms \\
\hline 8 & Learning how to play attentively & Helping the child to stay attentive when playing \\
\hline
\end{tabular}


consultation for the preschool teacher are provided (Booster TASH).

\section{Parent management and preschool teacher training (PMPTT)}

For children with no or only partial response to TASH, who are randomised to the PMPTT group, ageappropriate individually tailored behaviour therapy is provided in step 2. The 6-month PMPTT encompasses (1) parent management training, including parent-child interaction training, (2) preschool-teacher-focused interventions, including psychoeducation and behavioural interventions in the preschool, and (3) child-focused interventions (see Table 3). The primary goal of PMPTT is to reduce child problem behaviour and to enhance the parent-child and teacher-child relationship by improving parents' skills and teachers' educational skills. A total of 20 weekly sessions are provided. Parent- and teacher-focused interventions are based on the THOP [42] and on the PEP (Prevention Programme for Externalising Problem Behaviour; Präventionsprogramm für Expansives Problemverhalten - PEP [44]). Child-focused interventions are based on the Therapy Programme for the Improvement of Organisational Skills, Concentration and Impulse Control in Children with ADHD (Therapieprogramm zur Steigerung von Organisationsfähigkeit, Konzentration und Impulskontrolle

Table 3 Overview of the Parent Management Preschool Teacher Training (PMPTT) contents

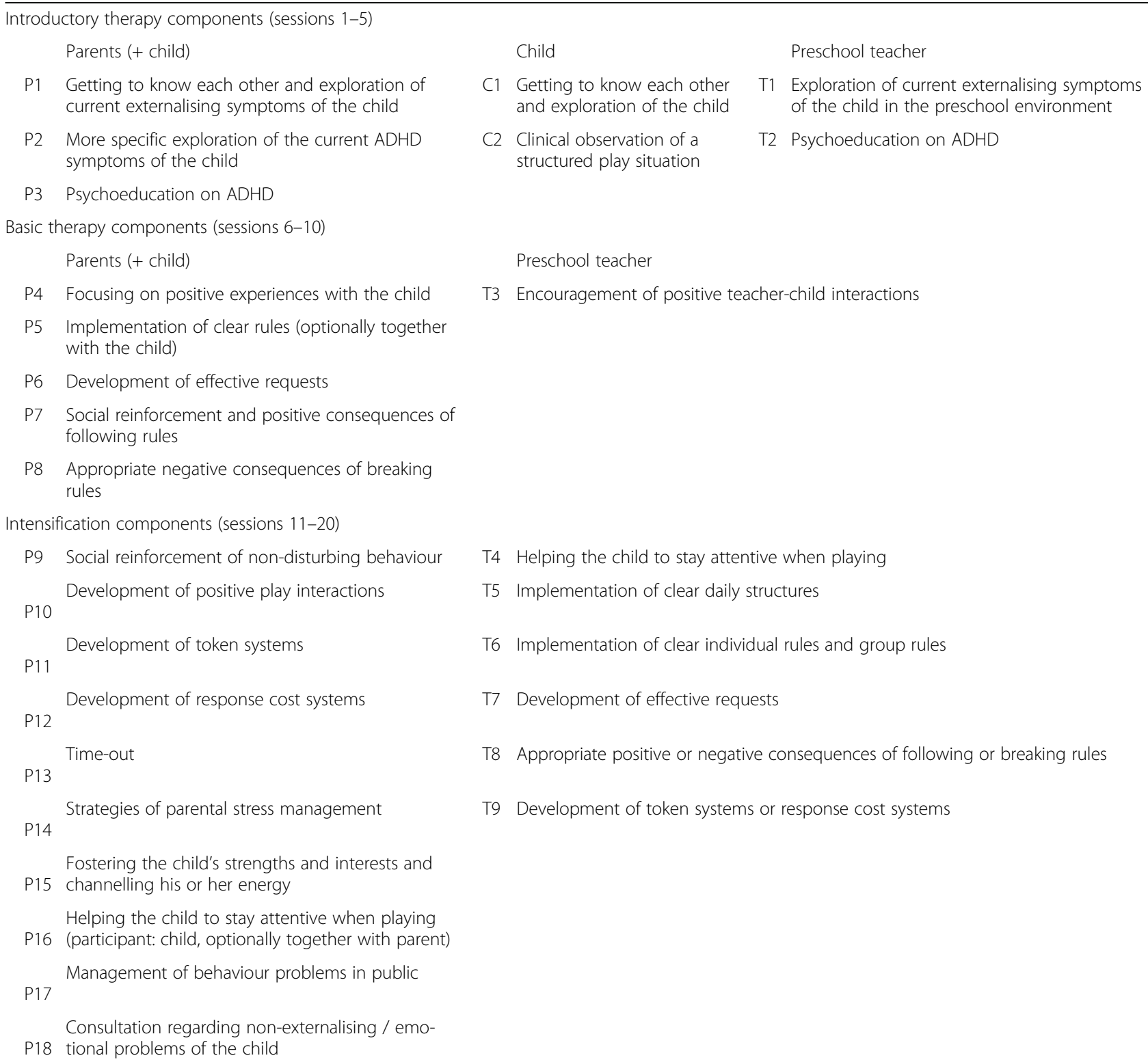

Child

P1 Getting to know each other and exploration of current externalising symptoms of the child

P2 More specific exploration of the current ADHD symptoms of the child

P3 Psychoeducation on ADHD

Basic therapy components (sessions 6-10)

Parents (+ child)

P4 Focusing on positive experiences with the child

P5 Implementation of clear rules (optionally together with the child)

P6 Development of effective requests

P7 Social reinforcement and positive consequences of following rules

P8 Appropriate negative consequences of breaking rules

Intensification components (sessions 11-20)

P9 Social reinforcement of non-disturbing behaviour P10 Development of positive play interactions

P11 Development of token systems Development of response cost systems P12

C1 Getting to know each other and exploration of the child

C2 Clinical observation of a structured play situation

Preschool teacher

T3 Encouragement of positive teacher-child interactions

T4 Helping the child to stay attentive when playing

T5 Implementation of clear daily structures

T6 Implementation of clear individual rules and group rules

T7 Development of effective requests

T8 Appropriate positive or negative consequences of following or breaking rules

T9 Development of token systems or response cost systems

\section{Exploration of current externalising symptoms of the child in the preschool environment}

T2 Psychoeducation on ADHD 
bei Kindern mit ADHS - THOKI-ADHS [45]). THOP is the only German treatment programme for ADHD with established efficacy [42]. PEP has been extensively evaluated in several trials with preschool children with externalising problem behaviour [9]. The PEP programme was originally developed for the training of parents and teachers of preschool children in a group format. For ESCApreschool, PEP materials were adapted for use in an individual format. PMPTT is conducted by clinical therapists who are trained during a 2-day workshop (see section on Treatment integrity).

\section{Treatment as usual (TAU)}

The other group of partial or non-responders to TASH receives TAU in step 2 , that is, a typical, mostly guideline-based ADHD preschool intervention. TAU is usually conducted by the participating centres but may also be performed by local cooperating institutions (e.g. child guidance centres, ergo-/occupational therapy, child and adolescent psychiatrists, psychotherapists). In the latter case, members of the cooperating institutions are asked to provide information about their treatment. To achieve a minimum of conformity, at least four patient contacts within the treatment period of 6 months are recommended.

Starting or optimising an additional ADHD pharmacotherapy (according to the clinical decision of the treating physician) is permitted in step 2 in the TAU condition as well as in the PMPTT condition, but has to be documented.

\section{Treatment integrity}

Treatment integrity is established through qualification standards for therapists (therapists have completed a university degree qualifying for training to become a licensed child and adolescent therapist, and are currently in training for psychotherapy with children and clinical expertise in the treatment of ADHD), study-specific therapist training, the use of the manualised treatment programmes and the use of protocol sheets for treatment documentation. TASH and PMPTT treatments are supervised by senior supervisors to check for adherence to the manual and study procedures. PMPTT therapists participate in three supervision sessions per patient. These sessions are scheduled after therapy sessions 5, 10 and 15, and are conducted either face to face or by telephone. For each patient, at least two video sequences are discussed with the supervisor. The TASH consultations are recorded in audio files and supervised regularly.

\section{Informants}

ESCApreschool collects information from different perspectives and informants: unblinded clinician (e.g. therapist or TASH counsellor), blinded clinician, the participating parent, the other parent/partner of participating parent, and, optionally, the preschool teacher.

The blinded clinicians conduct the clinical interviews with the parents, and are blind to the study condition. However, in order to minimise heterogeneity in ratings caused by different assessors, the same clinician should perform the interviews with a family at the different assessment time points. Therefore, the blinded clinicians are not blind to the assessment time point.

Additionally, to control for inter-rater reliability, the ratings of the parent interviews are audio- or videotaped. A clinician who is blind to both the treatment condition and the assessment time point rates a random selection of these interviews. The parent is the biological parent or guardian of the child, who is involved in the treatment (he or she is therefore not blind to the treatment condition). If possible, ratings by the child's preschool teacher are obtained.

\section{Measures \\ Main assessment time points}

Unless otherwise stated, the primary and secondary outcome measures are assessed at all four main assessment time points (T0/T1, T2, T3, T4). Figure 2 gives an overview of the measures assessed at the different time points.

Additional file 2: Figure S1 presents the time schedule in greater detail, with an overview of outcome measures, predictors and eligibility criteria.

\section{Primary and secondary outcome measures Primary outcome measures}

The primary outcome is the change in the combined ADHD and ODD symptom score. This combined symptom score is derived from the blinded clinician-rated ADHD and DBD Symptom Checklists based on a parent interview (DCL-ADHS + SSV Parent [46]). The DCLADHS and the DCL-SSV assess symptoms of ADHD or ODD and conduct disorder, respectively, according to DSM-5 and ICD-10 criteria. The DCL-ADHS consists of 18 items assessing ADHD symptoms and five items assessing functioning and psychological strain. The items on ADHD symptoms can be aggregated into two scales, inattention (nine items) and hyperactivity/impulsivity (nine items). The DCL-SSV comprises 28 items belonging to four subscales: $O D D$ (eight items), aggressive-dissocial problem behaviour (seven items), limited prosocial emotionality (11 items), and disruptive mood disorder (five items, three of which are also part of the $O D D$ scale). For ESCApreschool, we excluded the scale assessing disruptive mood disorder, as the associated symptoms are uncommon in preschool children. Moreover, the DCL-SSV includes five items on functioning and psychological strain. All items are rated on a 4-point Likert- 


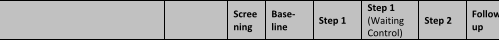
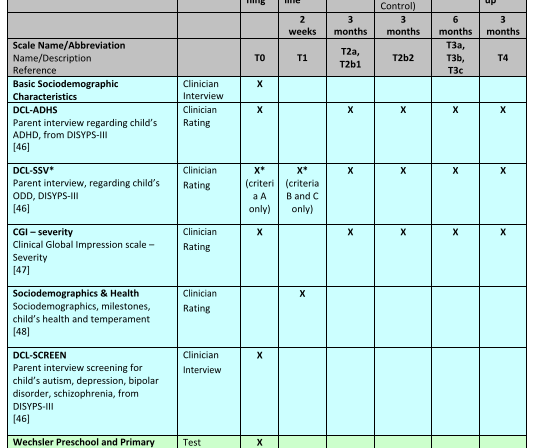 \\ Wechsler rerschool and priman
ccale of Intellizence

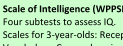 $\alpha>.68[46,63]$. ments in this age group. \\ Secondary outcome measures}

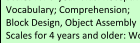
Scales for 4 years and older:
Reasoning, vocabulary, Mat
Reasoning, block Design Reasso
Reas
[49]
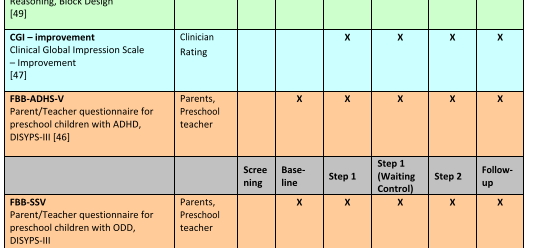

DISYPS-III
[46]

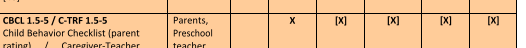

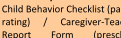

Report Form (prescht
tacher rating)
[50]

I50)
Ifrom 72 onwerds, only the
interal problems score will be

Internal priduras
assuessed]
cu-prescho

Parent Ras
Callous-U

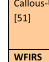

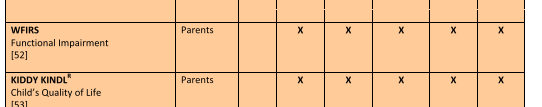

KLDDY KINOL"
Chil's Qually of Life
[5s]

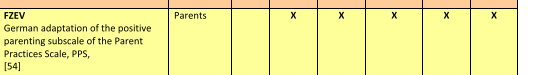

[54]

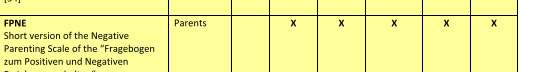

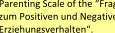

Erziehu
$[55]$
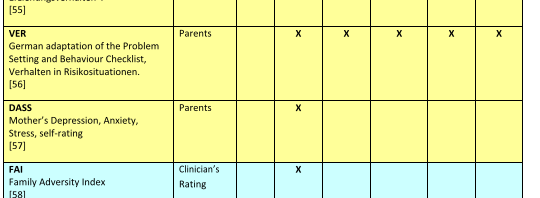

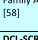
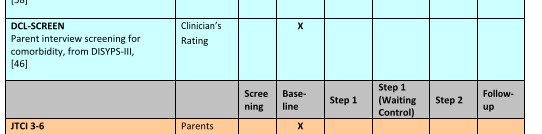

TrCl 3.6
Three subscales of the Junior
TTemperament and Character

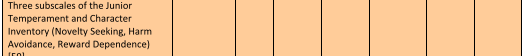

\begin{tabular}{|l}
\hline [59] \\
\hline SRS - short \\
\hline
\end{tabular}

SRS- - short
short version of the German
addentation

Short version of the Gert
adapation of the Socal
Responssveness scale

Respons
$[60,61]$

\begin{tabular}{l}
\hline $\begin{array}{l}\text { ADHS-SB/HASE } \\
\text { Mother's AOHD, self-rating } \\
\text { [62] }\end{array}$ \\
\hline OAA
\end{tabular}

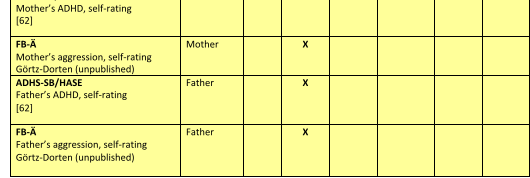

$1_{B \text { Blue rows refer to a clinical diagnosis based on a standardized interview, green rows to }}$

tests, orange rows refer to parent- or teacher-rated questionnaires, and yellow rows
concern questionnaires which aim to assess parents's personality and behaviour; thes

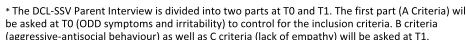

Fig. 2 Overview of measures and assessment time points type scale ranging from 0 to 3 , with higher scores indicating higher symptom severity. Scale scores are computed by averaging the associated item scores. The DCL-ADHS and the DCL-SSV subscales and total scores show satisfactory internal consistency (Cronbach's

We use a combined score of ADHD and ODD, since the two conditions are highly correlated in preschool children with ADHD, and the reduction of symptoms of ADHD and ODD is usually the main objective of treat-

The secondary outcome measures assess (1) ADHD and DBD symptoms as rated by parents and teachers, (2) psychosocial impairment, (3) comorbid symptoms and comorbid mental disorders, (4) quality of life of the child as perceived by parents, (5) social reactivity, and (6) parenting behaviour. All instruments used to assess symptoms and impairment are recommended in diagnostic guidelines for ADHD [12] and are employed in clinical practice. All secondary outcome measures have been validated both in English and in German and have been used in previous trials of pharmacological and psychotherapeutic interventions as well as in prevention studies. They have been widely used in children, and especially in preschool children with ADHD. All instruments provide population-based norms and therefore enable the calculation of age- and gender-adjusted normalisation rates.

\section{ADHD and DBD symptoms}

Parents and preschool teachers rate the symptom severity of $\mathrm{ADHD}$ and $\mathrm{DBD}$ on the ADHD Parent and Teacher Rating Scale for Preschool Children (FBBADHS-V; German: Fremdbeurteilungsbogen für Vorschulkinder mit Aufmerksamkeitsdefizit-/Hyperaktivitätsstörungen [46]) and on the DBD Parent and Teacher Rating Scale (FBB-SSV; German: Fremdbeurteilungsbogen für Störungen des Sozialverhaltens). The scales capture symptoms of ADHD or DBD, respectively, according to ICD-10 and DSM-5. The FBB-ADHS-V consists of 19 items belonging to the subscales inattention and hyperactivity/impulsivity. In the version for younger children (up to the age of 11), the FBB-SSV comprises 27 items, which can be aggregated into four subscales: $O D D$, aggressive-dissocial problem behaviour, limited prosocial emotionality, and disruptive mood disorder. All items are rated on a 4-point Likert-type scale ranging from 0 to 3 , with higher scores indicating higher symptom severity. Scale scores are computed by averaging the associated item scores. The subscales and the total score of both the FBB-ADHS and the FBB-SSV have demonstrated reliability and factorial validity. 
Clinical global impression and functional impairment The clinician-rated Clinical Global Impression Scale (CGI) is administered using a short clinical interview [47]. It is a widely used outcome parameter in clinical trials, measuring disease severity (CGI-severity, CGI-S) as well as general improvement during treatment (CGIimprovement, CGI-I). Both the CGI-S and the CGI-I are rated on a 7-point scale, with higher scores indicating greater severity or improvement, respectively. The CGI shows a good inter-rater reliability (.65-.92 [64];) and an intra-class correlation coefficient of .91 [65].

A German version of the parent-rated Weiss Functional Impairment Rating Scale, which was modified and adapted for use in preschool-age children, is applied to measure functional impairment $[66,67]$. The modified German version consists of 40 items, which are rated on a 4-point Likert-type scale ranging from 0 to 3 . Higher scores indicate greater impairment. The subscales and total score of the original version and of the modified German version have shown internal consistency $(\alpha>80)$ and factorial validity [66-68]. Moreover, the original version has demonstrated test-retest reliability, convergent validity, and responsiveness to change [68].

\section{Comorbid internalising and externalising symptoms}

The German version of the parent-rated Child Behavior Checklist 11/2-5 (CBCL 11/2-5 [69];; original English version: [70]) and the preschool teacher-rated CaregiverTeacher Report Form 11/2-5 (C-TRF 11/2-5; Arbeitsgruppe Deutsche Child Behavior Checklist, 2002b; original English version: [70]) are questionnaires to assess behavioural problems, emotional problems and somatic complaints of toddlers and preschool children aged $1 \frac{1 / 2}{2}$ to 5 years. Both scales comprise 99 items (83 overlapping items) rated on a 3-point scale ranging from 0 to 2 . The items can be aggregated into several syndrome scales as well as into three superordinate scales: internalizing problems, externalizing problems, and total problems. These superordinate scales are considered in ESCApreschool. For both the CBCL and the C-TRF, these scales have demonstrated satisfactory internal consistencies $(\alpha>.80)$ in German samples [69]. Moreover, analyses in German samples have provided evidence for the construct validity of the CBCL as well as limited evidence for the factorial validity of the CBCL internalizing problems and externalizing problems scales [69].

\section{Quality of life}

In evaluating health care with respect to prevention and treatment, quality of life has emerged as an important concept. We use the Kiddy-KINDL [53, 71, 72] to assess subjective generic health-related quality of life in preschool children. Results on the reliability of this scale vary: In a sample of preschoolers aged 4-6years,
Cronbach's alpha for the total score varied from .66 to .70 depending on age and gender [72]. However, in a larger sample of 3-6-year-old children, a Cronbach's alpha of .82 was reported [73].

\section{Callous-unemotional traits and social responsiveness}

Callous-unemotional traits are assessed with five items of the prosocial behaviour scale and the peer problems scale of the Strengths and Difficulties Questionnaire (SDQ [74]) and three items of the callous-unemotional dimension of the Antisocial Process Screening Device (APSD-CU [75], which were combined into a joint measure. The items are rated on a 3-point scale ranging from 0 to 2. Pasalich et al. [51] also combined items of the SDQ and the APSD, but used more items. They found satisfactory internal consistencies across mother, father and child ratings of their measure (range of $\alpha=$ 0.69-0.87).

Social responsiveness is assessed using a shortened 16-item version of the Social Responsiveness Scale (SRS-short [76]). The items of this questionnaire are rated on a 4-point scale ranging from 0 to 3 . The long version of the SRS shows high internal consistency $(\alpha=0.91-0.97)$ as well as satisfactory testretest reliability, inter-rater reliability, convergent validity and discriminative validity [60].

\section{Parenting behaviour}

Positive parenting behaviour is assessed using a German questionnaire covering positive parenting skills (Fragen zum Erziehungsverhalten, FZEV; Naumann, Kuschel et al., 2007). This questionnaire comprises 13 items rated on a 4-point scale ranging from 0 to 3, with higher scores indicating more positive parenting behaviour. The scale shows satisfactory internal consistency (mother ratings: $\alpha=0.85$; father ratings: $\alpha=0.87$ [77]). For the assessment of negative parenting behaviour, we apply the respective 13-item scale of the Questionnaire on Positive and Negative Parenting Behaviour (German: Fragebogen zum positiven und negativen Erziehungsverhalten, FPNE [55]). The items of this scale are rated on a 4-point scale ranging from 1 to 4 , with higher scores indicating more negative parenting behaviour. The scale is internally consistent $(\alpha=0.78)$.

Additionally, to assess parents' perceived sense of competency concerning challenging parenting situations, we employ a modified German Version of the Problem Setting and Behaviour Checklist (German: Verhalten in Risikosituationen, VER [77]). The 27 items of this questionnaire are rated on a 4-point scale ranging from 1 to 4 , with higher scores indicating a stronger sense of competency. The scale has demonstrated high internal consistency (mother ratings: $\alpha=0.92$, father ratings: $\alpha=0.94$ [77]). 


\section{Treatment satisfaction}

For the assessment of satisfaction with the treatment, treatment-specific parent satisfaction questions were developed (e.g. for the assessment of satisfaction with TASH). These are assessed as part of the clinical interview at $\mathrm{T} 2$ and $\mathrm{T} 3$.

\section{Feasibility and adherence measures}

Besides the outcome measures used at all of the main assessment time points ( $\mathrm{T} 1$ to $\mathrm{T} 4$ ), the following measures are used to assess feasibility and adherence:

(1) Clinical Feasibility Rating Scale (newly developed) to rate the feasibility of the TASH and PMPTT interventions (at $\mathrm{T} 2$ and $\mathrm{T} 3$ );

(2) Clinical Adherence Rating Scale (newly developed) to assess the adherence of the patient, the parents and preschool teachers during the interventions (at T2 and T3).

\section{Assessment of potential moderators of treatment response}

The following potential moderators of treatment response are analysed: (1) age, (2) gender, (3) socioeconomic status, (4) ADHD symptom severity, (5) comorbid symptoms, (6) intelligence, (7) parental depression, anxiety and stress (Depression, Anxiety and Stress Scale, DASS; Cronbach's $\alpha=0.89-.96$, test-retest reliability $r=$ 0.71-.81, validity [57, 78]), and (8) parental ADHD (ADHD Self-Rating Scale, German: ADHS-Selbstbeurteilungsskala, ADHS-SB; test-retest reliability $r=0.78-.89$; Cronbach's $\alpha=0.72-.9$, validity $[62,79])$.

\section{Psychometric data}

The following variables are assessed using a clinical interview with the parent during the diagnostic assessment at $\mathrm{T} 0$ and $\mathrm{T} 1$ and considered as possible predictors of treatment outcome: sociodemographic data of the child and the parent (e.g. child age, educational level of the parent or guardian), data on early child development (six items), temperament (13 items; Junior Temperament and Character Inventory [JTCI] 3-6 [59]), irritability (seven items; Affective Reactivity Index [ARI-Parent] [80]), and life events (14 items). Additionally, we employ the German version of the Family Adversity Index (FAI), adopted from the German Mannheimer Elterninterview [81]; original English version: [82, 83]). To measure parental aggression, we use the anger control scale of the German Elternfragebogen zum Umgang mit Ärger (FB- ̈̈) [Götz-Dorten, A. (unpublished) 2013; Department of Child and Adolescent Psychiatry, Psychosomatics and Psychotherapy, University of Cologne], which is a modified version of the 12-item form of the Aggression Questionnaire $[84,85]$. The clinical checklist Diagnose-
Checkliste zum Screening psychischer Störungen (DCLSCREEN; taken from the DISYPS-III [46]) is used to assess comorbid symptoms of depression (seven items), anxiety (ten items), autism spectrum disorder (four items), other neurodevelopmental disorders (six items), obsessive-compulsive disorder (two items) and tic disorders (one item). Based on a modified questionnaire by Piacentini et al. [86], the therapists additionally report their expectation of treatment benefit (three items) for a family. Moreover, the participating parents provide information about their own treatment expectations.

Furthermore, after every therapy session of step 1 and step 2, the therapist rates the treatment integrity (13 self-developed items), the treatment adherence of the client (ten items; eight items for TASH only), and current ADHD symptoms of the child (four items; shortened version of the German ADHD Questionnaire [87].

At the beginning of the study (T0/T1), children complete four subtests of the Wechsler Preschool and Primary Scale of Intelligence (WPPSI-III) to assess their IQ [49]. The 3-year-old children work on the scales Receptive Vocabulary, Comprehension, Block Design and Object Assembly, while the 4-6-year-olds complete the subtests Word Reasoning, Vocabulary, Matrix-Reasoning and Block Design.

\section{Biological data}

Transcranial sonography (TCS) is used to assess biological predictors of treatment response, especially in younger children. This is part of the transversal project ESCAbrain, which assesses biological data in all ESCAlife trials (see also [37-39]). Using TCS, the size of the echogenic region of the substantia nigra is assessed. In children, ADHD-associated hyperechogenicity of the substantia nigra has consistently been reported and has previously been identified as a potential biological marker of ADHD [88, 89]. TCS is a non-invasive method for the visualization of deep brain structures, such as the substantia nigra, through the intact skull. Ultrasound waves are reflected depending on tissue composition, resulting in different echogenicity of nuclei and ventricular system [38]. The method has no harmful side effects. Of particular interest is the mesencephalic scanning plane, including brainstem, substantia nigra and raphe nuclei. In terms of clinical implications, TCS can aid differential diagnosis (e.g. in movement disorders [90]) and has shown promise in predicting treatment response in psychiatric disorders in adult patients. As yet, no study has explored whether TCS can predict the effectiveness of non-pharmacological interventions. TCS is optional for the patients. As a well-tolerated investigation, it offers fast, non-invasive, targeted imaging in this age group in which magnetic resonance imaging (MRI) is not feasible or practical. 
Furthermore, saliva samples are collected before step 1 (T0/T1) and after step 2 treatment (T3) to determine predictive genetic and epigenetic patterns. These saliva samples are collected according to standard protocol of the ESCAmark subproject, which coordinates biosampling across the ESCAlife consortium. Samples are stored at $-80{ }^{\circ} \mathrm{C}$ and will be analysed after recruitment closure of all RCTs within ESCAlife. Samples will be used according to the ethics vote and data management plan of ESCAmark that will be published separately.

\section{Randomisation procedure}

Central randomisation with a 1:1 treatment ratio, analogous to the other ESCA trials [37-39], is performed by the CTU at the University Medical Centre Freiburg via fax, using block randomisation with variable block length to ensure concealment of randomisation. Randomisation is stratified by centre. The randomisation request form contains the study-specific patient identification number, year of birth and the confirmation of ADHD above the cut-off. The CTU reviews the patient's details on the randomisation fax and performs the randomisation if the data on the fax are appropriate and complete.

\section{Quality assurance and monitoring}

The monitoring is performed by the clinical research associates (CRAs) of the CTU. Adapted monitoring is accomplished according to Good Clinical Practice (ICHGCP E6) and standard operating procedures (SOP). This verifies that patients' rights and well-being are protected, that reported trial data are accurate, complete and verifiable from source documents, and that the trial is conducted in compliance with the currently approved protocol/amendment, with GCP and with the applicable regulatory requirements to ensure safety and integrity of clinical trial data. In this trial, all trial-specific monitoring procedures, monitoring visit frequency and the extent of source data verification (SDV) are predefined in a specific monitoring manual.

The investigator accepts monitoring visits before, during and after the clinical trial. Prior to the trial, a pretrial telephone consultation and a site initiation visit at each site are conducted in order to train and introduce the investigators and their staff to the trial protocol, essential documents and related trial-specific procedures, ICH-GCP and national/local regulatory requirements.

During the trial, the monitor visits the site regularly depending on the recruitment rate and quality of data. During these on-site visits, the monitor verifies that the trial is being conducted according to the trial protocol, trial-specific procedures, ICH-GCP and national/local regulatory requirements. Moreover, the monitor checks that signed informed consent has been provided, and verifies the eligibility of patients, completeness of primary endpoint questionnaires, treatment compliance, and documentation. The monitor also performs source data verification to ensure that clinical trial data are recorded and documented in the source data and that case report forms (CRFs) are complete and accurate. In the case of data quality problems or a high number of protocol violations at individual sites, the extent of source data verification and frequency of monitor visits is adapted accordingly.

The investigator must maintain source documents for each patient in the trial, consisting of case and visit notes (hospital or clinic medical records) containing demographic and medical information, laboratory data, and the results of any other tests or assessments. All information recorded on CRFs must be traceable to source documents in the patient's file. The investigator must also keep the original signed informed consent form (a signed copy is given to the patient).

The investigator must give the monitor access to all relevant source documents to confirm their consistency with the CRF entries.

An independent Data Monitoring Committee (DMC), composed of Prof. Dr. H. J. Freyberger, Prof. Dr. A. Rothenberger and Prof. Dr. J. Schmitt, advises the trial sponsor on patient safety and measures to ensure the credibility and integrity of the ongoing trial.

\section{Stopping rules}

Inclusion in the study is not possible without written informed consent of parents/guardians. If a preschool child requires inpatient treatment or needs a different kind of treatment for health reasons according to the judgment of the attending physician, the child will be excluded from the study. Study exclusion will also occur if any other factors arise which affect the child's wellbeing. The Ethics Committee will be informed immediately in the case of severe events during the conduct of the trial. Global stopping rules for the trial or closing of a centre include the emergence of data leading to a revision of the risk-benefit ratio, ongoing failure of recruitment, or repeated violations of standard GCP rules or of the study protocol. For a decision on the termination of the trial or on closing a participating centre, agreement between the study coordinator, PIs, site investigators, DMC members, the responsible Ethics Committee and the CTU Freiburg is intended.

\section{Sample size and power calculations}

The whole stepped care design is primarily powered for the two RCTs in step 1 and step 2. Based on the results of previous studies, an effect size of $d=0.5$ is expected for the RCT in step 1 (TASH compared to a waitlist control group). Kierfeld et al. [30] found a moderate to 
large effect $(d=0.79)$ of a TASH intervention compared to a waitlist control group on parent-rated ADHD symptoms. Both the intervention and the outcome measure were similar to those used in this current trial. Effect sizes of about SMD $=0.75$ were found for parent management training interventions, mainly compared to waiting groups in children with disruptive behaviour problems, using unblinded parent ratings [18]. However, reported effects sizes are smaller when blinded ratings are applied [23]. Therefore, we expect an effect size of $d=0.5$ for the primary outcome for the randomisation comparing PMPTT with TAU in step 2.

The calculation of the sample size (software: STPLAN Version 4.3) is based on the primary endpoint of step 2 (ADHD/ODD change score from T2 to T3). Using a two-sided $t$ test with a power of $80 \%$ at a significance level of 5\%, 64 patients with non-missing data per group are required to detect a difference when the true effect size is $d=0.5$. To account for the possibility that some patients (10\%) will have incomplete data at T3, in total, 144 partial or non-responders should be randomised at step 2. We assume that about $20 \%(n=36)$ will show a full response after step 1 [30] and that a group of $10 \%$ will have dropped out from T1 to T2. Therefore, 180/ $0.9=200$ patients should be randomised at T1 (step 1). Given 180 patients with complete data and a presumed true effect size of $d=0.5$, the power to detect a difference is $92 \%$. We assume that about 300 patients will need to be screened for study participation.

\section{Statistical analyses}

Before the inclusion of the first patient, a detailed statistical analysis plan (SAP) was prepared. This will be completed during the 'blind review' of the data, at the latest. If the SAP contains any changes to the analyses outlined in the trial protocol, they will be marked as such, and reasons for amendments will be given.

All statistical programming for analysis will be performed with the Statistical Analysis System (SAS Institute, Cary, NC, USA).

\section{Definition of populations included in the analyses}

The primary analysis will be conducted according to the intention-to-treat (ITT) principle. This means that the patients will be analysed in the treatment arms to which they were randomised, irrespective of whether they refused or discontinued the treatment or whether other protocol violations become apparent.

The per-protocol (PP) population is a subset of the full analysis set (FAS) and is defined as the group of patients who had no major protocol violations, received a predefined minimum dose of the treatment and underwent the examinations required for the assessment of the endpoints at relevant, predefined times. The analysis of the
PP population will be performed for the purpose of a sensitivity analysis.

Safety analyses will be performed in the safety population. Patients in the safety population are analysed as belonging to the treatment arm defined by treatment received. Patients are included in the respective treatment arm if treatment was started/if they received at least one dose of trial treatment.

\section{Patient demographics/other baseline characteristics}

Demographic and other baseline data (including disease characteristics) will be summarised descriptively using all documented patients. Continuous data will be summarised by arithmetic mean, standard deviation, minimum, $25 \%$ quantile, median, $75 \%$ quantile, maximum, and the number of complete and missing observations. If appropriate, continuous variables can also be presented in categories. Categorical data are summarised by the total number of patients in each category and the number of missing values. Relative frequencies are displayed as valid percentage (number of patients divided by the number of patients with non-missing values).

\section{Analysis of primary endpoint}

The primary statistical analyses of steps 1 and 2 will be by ITT, that is, all randomised patients will be analysed according to their allocated arm. Changes in the DCL ADHD + ODD (DBD) Parent scores between T1baseline and T2 (after TASH/waiting) or T2 (after TASH) and T3, respectively, will be evaluated in separate mixed-effects models for repeated measures (MMRM). The MMRMs will include fixed categorical effects of treatment, centre, visit and treatment-by-visit interaction, and continuous, fixed covariates of baseline and baseline-by visit interaction. Further covariates predictive of missingness will be included based on a prespecified selection strategy, to correct for potential bias arising from missing data.

Unstructured covariance matrices will be used to model within-patient correlations. The primary treatment comparisons of the change scores at T2 and T3 will be based on least-squares means with two-sided 95\% confidence intervals without correction for multiple testing. Other possibly relevant covariates may be considered as well. Subgroup analyses will be conducted in an exploratory manner by including interaction terms in the MMRMs. These will focus on the analyses of patients' and parents' comorbidity. In addition, gender effects will be investigated as prognostic and predictive factors. Exploratory within-subjects comparisons (change in step 1 compared to change in step 2) will also be also carried out in MMRMs. Secondary efficacy endpoints derived from other scale scores will be analysed in the same manner, i.e. with the same type of linear 
model. Follow-up of full responders after step 1 will be evaluated descriptively. No interim analysis for efficacy will be performed.

Safety/tolerability analyses will be carried out in all patients for whom one of the randomised treatments was started, according to treatment received.

\section{Analysis of secondary endpoints}

Secondary endpoints will be analysed descriptively in a similar fashion to the primary outcome. Scores will be calculated according to the respective manuals.

The analysis of the change between T1-baseline and T2 (after TASH/waiting) and T3 (after TAU/PMPTT) comprises the analysis of the primary endpoint for continuous measurements (DCL-ADHS, DCL-SSV, FBBADHS, FBB- SSV, CU-Preschool, CBCL/1,5-5, CTRF, WFIRS, KIDDY KINDL, FZEV, FPNE, VER). In addition, for DCL-ADHS and the measurements listed above, change between $\mathrm{T} 2$ and $\mathrm{T} 3$ in patients without ADHD/ ODD (at T2) will be evaluated. Treatment effects will be calculated with two-sided $95 \%$ confidence intervals.

The within-patient changes between T2 and T3 in patients without ADHD/ODD (at T2) in continuous endpoints will be analysed using linear regression adjusted for the baseline measurement and study centre.

The difference in the CGI between T1 and T2 and between T2 and T3 in the randomised steps will be analysed using the Mann-Whitney $U$ test. The withinpatient difference in the CGI will be analysed using the Wilcoxon signed-rank test.

Possible predictors of the DCL-ADHD score will be analysed using linear regression.

\section{Legal and ethical foundation}

Before trial start, all relevant documents were submitted to the local Ethics Committee responsible for the respective participating centres. The primary vote on the study was obtained from the Ethics Committee of the Medical Faculty of the Philipps University of Marburg. For changes to the trial protocol that are formal in nature or include relevant changes for participants, the ethics committees have to vote anew.

\section{Discussion}

ESCApreschool (investigating 3-6-year-old preschool children with ADHD) is one part of the multicentre study ESCAlife, which examines clinical care for children, adolescents and adults with ADHD to optimize evidence-based personalised stepped care approaches across different age groups (see also [37-39]).

Early onset, high prevalence and persistence, and developmental comorbidity make ADHD a psychosocially impairing and cost-intensive mental disorder. Despite continuous treatment research, there is still a substantial need to optimise individualised treatment strategies in order to improve outcomes and reduce the economic burden. By covering the full spectrum of ADHD at all ages, the consortium will be able to make significant recommendations for improving ADHD treatment in routine clinical care.

Clinical guidelines recommend an adaptive treatment and a stepped care approach for the treatment of ADHD/ODD [12]. However, this approach has not yet been empirically validated. The main goal of ESCApreschool is therefore to assess the efficacy of a stepped care approach in children with ADHD/ODD aged 3-6 years and to identify predictors as well as moderators of treatment outcome. The design combines two RCTs. The first aims to analyse the efficacy of the lowthreshold TASH intervention by means of a randomised waiting control design and to identify predictors of response. Partial and non-responders to TASH take part in a second RCT, which compares the effects of an intense behaviour therapy addressing parents, children and preschool teachers with TAU. Thus, the design allows for the evaluation of the additional effects of behaviour therapy and TAU in preschool children with ADHD/ ODD who did not sufficiently respond to the lowthreshold intervention in step 1 of the study.

The results will improve future guidelines on the treatment of preschool-age children with ADHD and/or ODD. Moreover, the findings may also be used to develop usable, potentially more costeffective, individualised stepped care pathways for young children with ADHD/ODD. The evaluation of predictors of treatment response will help to identify indications for specific treatments during the therapy process. Resource-intensive therapeutic interventions can then be specifically directed at individuals who will probably not respond to or benefit from low-threshold treatment. This would help to distribute resources efficiently and improve treatment for preschool psychiatric patients. Such a targeted, resource-friendly use of interventions will help children with ADHD, their families, preschool teachers, peers, the health care system and society as a whole.

\section{Trial status}

The trial was registered at the German Clinical Trials Register (DRKS) as a Current Controlled Trial under DRKS00008971 on 1 October 2015. This manuscript is based on protocol version 3 (14 October 2016). Recruitment started January 2016 with the first patient enrolled on 29. June 2016 (first patient in). The milestone of 75\% (150 patients) was reached in June 2019. Recruitment for this trial is ongoing. Recruitment will be completed in approximately December 2019. 


\section{Supplementary information}

Supplementary information accompanies this paper at https://doi.org/10. 1186/s13063-019-3872-8

Additional file 1: Table S1. Overview of the telephone-assisted selfhelp (TASH) booklets for preschool teachers.

Additional file 2: Figure S1. Time schedule with an overview of outcome measures, predictors and eligibility criteria.

Additional file 3. SPIRIT 2013 Checklist: Recommended items to address in a clinical trial protocol and related documents.

\section{Abbreviations}

ADHD: Attention-Deficit/Hyperactivity Disorder; AKiP: School of Child and Adolescent Cognitive Behaviour Therapy [Ausbildungsinstitut für Kinder- und Jugendlichenpsychotherapie]; Booster SH: Booster Self-Help; CAP: Child and Adolescent Psychiatry; CBCL 11/2-5: Child Behaviour Checklist for Ages 11/2-5; CGI: Clinical Global Impression Scale; CRF: Case report form; C-TRF: Caregiver and Teacher Report Form of the Child Behaviour Checklist; CTU: Clinical Trials Unit; CUpreschool: Parent Rating Callous-Unemotional Traits (preschool age); DASS: Depression Anxiety Stress Scales; DBD: Disruptive behaviour disorder; DCL-ADHS: Clinical ADHD Checklist [Diagnose-Checkliste für Aufmerksamkeitsdefizit-/Hyperaktivitätsstörungen]; DCL-SCREEN: Clinical Checklist for Screening Mental Disorders [Diagnose-Checkliste zum Screening psychischer Störungen]: DCL-SSV: [Diagnose-Checkliste für Störungen des Sozialverhaltens]; DGKJP: German Association for Child and Adolescent Psychiatry, Psychosomatics and Psychotherapy [Deutsche Gesellschaft für Kinder- und Jugendpsychiatrie, Psychosomatik und Psychotherapie]; DISYPSIII: Diagnostic System for Mental Disorders in Children and Adolescents Version III [Diagnostiksystem für psychische Störungen nach ICD10 und DSM-5 für Kinder und Jugendliche-III; DMC: Data Monitoring Committee; DRKS: German Clinical Trials Register [Deutsches Register Klinischer Studien]; DSM-5: Diagnostic and Statistical Manual of Mental Disorders, Fifth Edition, Classification and Diagnostic Tool for Mental Disorders issued by the American Psychiatric Association; ESCAadol: Evidence-based stepped care of ADHD in Adolescents (aged 12-17 years); ESCAlate: Evidence-based stepped care of ADHD in Adults (aged 16-45 years); ESCAlife: Evidence-based stepped care of ADHD along the Lifespan; ESCApreschool: Evidence-based stepped care of ADHD in preschool children (aged 3-6years); ESCAschool: Evidencebased stepped care of ADHD in school children (aged 6-12 years); FAl: Family Adversity Index; FAS: Full Analysis Set; FBB-ADHS: External Rating Questionnaire for ADHD [Fremdbeurteilungsbogen für Aufmerksamkeitsdefizit/ Hyperaktivitätsstörungen]; FBB-SSV: Self-Assessment Questionnaire for ADHD [Fremdbeurteilungsbogen für Störungen des Sozialverhaltens]; FPNE: Questionnaire on Positive and Negative Parenting Behaviour [Fragebogen zum positiven und negativen Erziehungsverhalten]; FZEV: Questionnaire on Parenting Behaviour [Fragen zum Erziehungsverhalten]; ICD-10: International Classification of Diseases, Tenth Edition; ICH-GCP: Good Clinical Practice; ID: Identifier; IQ: Intelligence quotient; ITT: Intention-to-treat; JTCl: Junior Temperament and Character Inventory; Kiddy-KINDL: Instrument for assessing subjective generic healthrelated quality of life in preschoolers; MMRM: Mixed-effects model for repeated measures; MRI: Magnetic resonance imaging; ODD: Oppositional defiant disorder; PEP: Prevention Programme for Externalising Problem Behaviour [Präventionsprogramm für Expansives Problemverhalten]; PI: Principal Investigator; PMPTT: Parent Management and Preschool Teacher Training; pp.: per protocol; RCT: Randomized controlled trial; SAP: Statistical Analysis Plan; SDQ : Strengths and Difficulties Questionnaire; SDV: Source Data Verification; SMD: Standardised Mean Difference; SRS-short: Social Responsiveness Scale, short version; T0: Screening at the beginning of the study (to assess inclusion and exclusion criteria); T0/T1: Indicates overlap in diagnostic procedure of T0 and T1 assessment; T1: Assessment at baseline before step 1; T2: Assessment at the end of step 1 and before the beginning of the step 2 intervention; T3: Assessment at the end of the step 2 intervention; T4: Follow-up assessment 3 months after the end of the step 2 intervention; TASH: Telephone-Assisted Self-Help for parents and preschool teachers; TAU: Treatment as usual; TCS: Transcranial sonography; THOP: Treatment Programme for Children with Externalising Disorders [Therapieprogramm für Kinder mit hyperaktivem und oppositionellem Problemverhalten]; VER: Questionnaire assessing behaviour in risk situations
[Verhalten in Risikosituationen]; WFIRS: Weiss Functional Impairment Rating Scale - Parent Report; WPPSI-III: Wechsler Preschool and Primary Scale of Intelligence, third edition

\section{Acknowledgements}

We thank all staff members who are participating in the ESCApreschoolstudy in nine different study recruiting centres and who are involved in recruitment, study organisation and management, blind rating, diagnostics and therapy as well as the ESCAbrain study group and the CTU.

These are (sorted by recruiting centre affiliation and in alphabetical order): Study centre Aachen: Lea Jahnen, Kerstin Konrad (PI), Vanessa Reindl Study centre Göttingen: Fatmeh Al-Debi, Luise Poustka (PI), Henrik Uebel-von Sandersleben, Jan Schulz

Study centre Hamm: Miriam Davids, Natalie Deux, Mareen Dökel, Silvia

Eißing, Regina Herdering, Carina Huhn, Lara Kaffke, Inken Kirschbaum-Lesch, Tanja Legenbauer, Franziska Martin, Nina Müller, Nicola Nolting, Daniela Pingel, Carina Schulz, Karen Schumann, Birthe Wagner, Daniela Walkenhorst Study centre Köln: Paula Altenberger, Ruth Beckmann, Caroline Betcher, Jana Bretthauer, Corinna Broekmans, Yvonne Buntrock, Mareike Bürger, Dominique Deli, Elvan Dogan, Katrin Floß, Anna Fronhofen, Nina Geldermann, Claudia Ginsberg, Jana Harenkamp, Julia Kellner, Claudia Kinnen, Marie-Theres Klemp, Hedda Körner, Constanze Kitzig, Katrin Krugmann, Jessica Leite, Sarah Meier, Judith Mühlenmeister, Lea Ostrowski, Jeannine Pawliczek, Daniela Perri, Judith Ratayczak, Stephanie Schürmann, Ellen Settegast, Johanna Spicher, Susanne Stollewerk, Ann-Kathrin Thöne, Laura Wähnke, Annabelle Warschburger, Friederike Waschau, Tanja Wolff Metternich-Kaizman, Sara Zaplana Labarga

Study centre Mannheim: Karina Abenova, Brigitta Gehrig, Monja Groh, Christine Igel, Anna Kaiser, Theresa Nickel, Angelina Samaras, Marie-Therese Steiner, Nina Trautmann, Matthias Winkler, Mirjam Ziegler

Study centre Marburg: Anette Becker, Anita Dehnert, Sabine Finkenstein, Charlotte Finger, Claudia Freitag, Nicole Grau, Wiebke Haberhausen, Julia Häusser, Daria Kasperzack, Franziska Körfgen, Jana Langhammer, Christopher Mann, Tanja Mingebach, Thomas Mooz, Jens Pfeiffer, Alisa Samel, Bastian Schrott, Anna Schuh, Isabell Schulz, Thomas Stehr, Rebecca Stein, Anne von Stiphout, Linda Weber, Maximiliane Werther

Study centre Neuruppin: Liane Adomat, Stefanie Bienioschek, Elisabeth Heukamp, Michael Kölch (PI), Anne Schüller

Study centre Tübingen: Yael Amling, Ute Dürrwächter, Anna Hertle, Natalie Herrmann, Melinda Mross, Anja Pascher, Alena Roth, Priska Schneider, Anja Schöllhorn, Ida Steinacker

Study centre Würzburg: Christoph Biohlawek, Lisa Eidenschink, Julia Geissler, Silke Hauck, Annette Nowak, Corina Otte, Isabel Paul, Katharina Peters Additional ESCAbrain staff members, who are involved in ESCApreschool: Clinical Trials Unit Freiburg: Veronika Frommberger, Alexander Hellmer, Lydia Herbstritt, Maria Huber, Stefanie Lade, Patrick Müller, Ralf Tostmann, Barbara Schilling, Carla Schneider

Further thanks to Udo Koenig for finalizing EndNote citations according to the author guidelines. Thank you to Sarah Mannion for English-language proofreading.

\section{Dissemination policy and access to data}

Results will be communicated to the public via presentations at scientific conferences and meetings, via publications in peer-reviewed scientific journals, via education courses in ADHD treatment, press releases and self-help organisations. PIs from all participating study sites and the CTU Freiburg, as well as any scientific staff members significantly contributing to an individual publication of data collected for ESCApreschool, are eligible for authorship. These persons will be granted access to the final dataset. Whether the dataset will be available after publication, and how, is not yet decided.

\section{Authors' contributions}

$\mathrm{KB}$ is $\mathrm{PI}$ of the ESCApreschool trial, developed the basic study design (together with MD) and wrote the grant application for ESCApreschool, is part of the ESCAlife-Consortium, is head of the Marburg recruiting centre for the ESCA children trials (ESCApreschool, ESCAschool and ESCAadol) and drafted the manuscript. TB coordinates the ESCAlife consortium and codeveloped the basic study design. He was involved in critically revising the manuscript for important intellectual content. DB made substantial contributions to the conception and design of the ESCAlife research proposal, and heads the sub-project ESCAbrain. He was involved in critically revising the 
manuscript for important intellectual content. CD supports MD in coordinating ESCApreschool at the Cologne study site and coordinated the TASH intervention from 2015 to 2017. She prepared the Tables 2 and 3 and Additional file 1: Table S1 of the current article, wrote parts of the sections on the TASH intervention and critically revised this manuscript. CH critically revised this manuscript. MH was involved in the development of the research proposal, is head of the Hamm recruiting centre and critically revised this manuscript. LJ and AKT coordinate ESCApreschool at the Cologne study site and contribute to the implementation of the study. They coordinate and provide supervision for PMPTT treatment and were involved in critically revising this manuscript. CJ is responsible for the statistical planning of the trial and wrote the sections on sample size and statistical analysis in this manuscript. KJ (until January 2017) and JK (since Febuary 2017) are scientific staff members at the Department for Child and Adolescent Psychiatry (CAP) in Marburg, are involved in the management and organisation of the study, and are responsible for recruitment as well as data acquisition. Both revised the final manuscript critically. JK added, together with AKW, Fig. 2. SM assists TB by coordinating the ESCAlife consortium and was involved in critically revising the manuscript. UPP made substantial contributions to the conception and design of ESCApreschool and has been involved in drafting the manuscript and revised it critically for important intellectual content. TR heads the Tübingen study site of ESCApreschool and contributed to the implementation of the study. He revised the draft and has approved all contents. MR and TJ are part of the ESCAlife Consortium, have contributed to the design of ESCApreschool and are responsible for the conduct of ESCApreschool at the Würzburg site. They were involved in critically revising this manuscript. EvW supports MD in coordinating ESCApreschool at the Cologne study site and contributes to the implementation of the study. She coordinates and provides supervision for PMPTT treatment and was involved in critically revising this manuscript. AKW is a scientific staff member at the Marburg study site, is responsible for agreements with the recruiting centres as well as additional important management tasks in ESCA. She provided support for Table 1 and Fig. 2, added the SPIRIT guideline checklist and revised the manuscript critically for important intellectual content. MD is co-principal investigator (Co-PI) of the ESCApreschool trial, developed the basic study design (together with KB), is part of the ESCAlife-Consortium, is head of the Cologne recruiting centre for the ESCA children trials (ESCApreschool, ESCAschool and ESCAadol) and critically revised the manuscript. All authors gave final approval of the last version of the manuscript and agreed to be accountable for all aspects of the work in ensuring that questions related to the accuracy or integrity of any part of the work are appropriately investigated and resolved.

\section{Authors' information}

KB is Chair for Child and Adolescent Psychiatry and Psychotherapy at the Philipps University in Marburg and Head of the Department for Child and Adolescent Psychiatry, Psychosomatics and Psychotherapy at the University Hospital Marburg.

TB is Professor of Child and Adolescent Psychiatry at the Medical Faculty Mannheim of the University of Heidelberg, Medical Director of the Department of Child and Adolescent Psychiatry of the Central Institute of Mental Health (CIMH), Deputy Director of the CIMH in Mannheim, Germany, and Chair of the European Network for Hyperkinetic Disorders (EUNETHYDIS). DB is Professor and Research Group Leader on Developmental Clinical Neurophysiology at the Department of Department of Child and Adolescent Psychiatry, CIMH, Mannheim, Germany and Professor at the Department of Child and Adolescent Psychiatry, Medical Faculty, University of Zurich, Switzerland and Head of Brain Mapping Research in Zurich.

$\mathrm{CD}, \mathrm{CH}$ and EvW are psychotherapists and senior researchers at the Department of Child and Adolescent Psychiatry, Psychosomatics and Psychotherapy, University of Cologne, Germany.

$\mathrm{MH}$ is Professor for Child and Adolescent Psychiatry at the Department for Child and Adolescent Psychiatry, LWL University Hospital Hamm at the Ruhr University Bochum, Germany and Head of the Department for Child and Adolescent Psychiatry, LWL University Hospital Hamm.

$\mathrm{TJ}$ is a senior psychologist, psychotherapist and researcher at the Department for Child and Adolescent Psychiatry, Psychosomatics and Psychotherapy at the University Hospital Würzburg.

$\sqcup$ and AKT are psychotherapists and researchers at the Department of Child and Adolescent Psychiatry, Psychosomatics and Psychotherapy, University of Cologne, Germany.
$\mathrm{CJ}$ is the trial statistician and works as Deputy Head of the Biometrics and Data Management Section at the Clinical Trials Unit - University Medical Centre Freiburg.

KJ, JK, UPP and AKW are scientific staff members at the Department for Child and Adolescent Psychiatry, Psychosomatics and Psychotherapy at the University Hospital Marburg and UPP is also Head of the Scientific Working Group Developmental Psychopathology.

SM is a scientific staff member at the Department of Child and Adolescent Psychiatry of the Central Institute of Mental Health $(\mathrm{CIMH})$ and helps TB to coordinate the ESCAlife-consortium.

TR is Professor for Child and Adolescent Psychiatry and Psychotherapy at the University of Tübingen and Head of the Department of Child and Adolescent Psychiatry and Psychotherapy, University Hospital Tübingen, Germany.

MR is Chair for Child and Adolescent Psychiatry and Psychotherapy at the Julius-Maximilians University in Würzburg and Head of the Department for Child and Adolescent Psychiatry, Psychosomatics and Psychotherapy at the University Hospital Würzburg.

MD is Professor for Psychotherapy in Child and Adolescent Psychiatry at the Department of Child and Adolescent Psychiatry, Psychosomatics and Psychotherapy, Medical Faculty of the University of Cologne, Germany, and Head of the School of Child and Adolescent Cognitive Behaviour Therapy (AKiP), University Hospital of Cologne, Germany.

\section{Funding}

ESCApreschool is funded by the German Federal Ministry of Education and Research (BMBF) Grant 01EE1408. This grant provided the financial means for study coordination, materials and compensating participating centres for study-associated additional workload, training, and presentation of the trials in meetings. The TASH intervention is funded, as this is so far not financed by the health insurance system. Standard ADHD/ODD routine diagnostic procedures (except for additional diagnostics as part of the study) as well as PMPTT and TAU are not funded. The funding source had no role in the trial design of this study and will play no role in its execution, analyses and interpretation of the data, or the decision to publish results.

\section{Availability of data and materials} Not applicable.

\section{Ethics approval and consent to participate}

The ESCApreschool study has been approved by the Ethics Committee of the Medical Faculty of the Phillips University of Marburg, Germany (No. 87/ 15). Additionally, it has been approved by all local Ethics Committees of the participating centres in Germany: Faculty of Medicine of the Ruhr-University Bochum (No. 15-5523), the Medical Faculty of the University of Cologne (No. 15-335), the Ethics Committee of the State Chamber of Medicine (Landesärztekammer) Baden-Württemberg (No. B-F-2015-080), the Medical Faculty of the University of Würzburg (No. 254/15_z), the Faculty of Medicine of the Eberhard Karls University Tübingen (No. 728/2015BO2), the Medical Faculty of the University Aachen (No. EK 204/17), the Medical Faculty of the University Göttingen (No. 4/3/17) and the Brandenburg Medical School Theodor Fontane.

In order to participate in the study, both parents/guardians are informed about the study procedures and goals and give their written informed consent. The preschool child has additionally to give his/her assent for participation.

\section{Consent for publication}

Not applicable.

\section{Competing interests}

KB has been involved in research/clinical trials with Eli Lilly $(\leq 2011)$ and Shire ( $\leq 2010)$, was on the Advisory Board of Eli Lilly/Germany ( $\leq 2014)$, a member of the Scientific Committee of Shire $(\leq 2012)$ and was paid for public speaking by Eli Lilly (<2011) and Shire (2015). These activities do not bias the objectivity of this manuscript (in her opinion), but are mentioned for the sake of completeness.

TB served in an advisory or consultancy role for Lundbeck, Medice, Neurim Pharmaceuticals, Oberberg GmbH, Shire, and Infectopharm. He received conference support or speaker's fees from Lilly, Medice, and Shire. He 
received royalties from Hogrefe, Kohlhammer, CIP Medien, and Oxford University Press.

DB served as an unpaid scientific advisor for an EU-funded neurofeedback trial unrelated to the present work.

$\mathrm{CD}$ and $\mathrm{MD}$ were involved in the development of the materials used in the TASH intervention and will receive royalties after publication of the materials. $\mathrm{MH}$ served in an advisory or consultancy role for Lilly, Shire, and Medice, and received conference attendance support or was paid for public speaking by Medice, Neuroconn, and Shire.

MD received consulting income and research support from Lilly, Medice, Shire, Janssen Cilag, Novartis, and Vifor. He received income as Head of the School for Child and Adolescent Behaviour Therapy at the University of Cologne and royalties from treatment manuals, books and psychological tests published by Guilford, Hogrefe, Enke, Beltz, and Huber, including the materials used in the PMPTT intervention.

$C H, T J, L J, C J, K J, J K, S M, ~ U P P, T R, M R, A K T$, EvW, and AKW are not aware of any biases that might be perceived as affecting the objectivity of this manuscript and declare that they have no competing interests.

\section{Author details}

'Department of Child and Adolescent Psychiatry, Psychosomatics and Psychotherapy, Medical Faculty of the Philipps-University Marburg, Hans-Sachs-Str. 6, 35039 Marburg, Germany. ${ }^{2}$ Center for Mind, Brain and Behavior (CMBB), University of Marburg and Justus Liebig University Giessen, Marburg, Germany. ${ }^{3}$ Department of Child and Adolescent Psychiatry and Psychotherapy, Central Institute of Mental Health, Heidelberg University, Medical Faculty Mannheim, Mannheim, Germany. ${ }^{4}$ Department of Child and Adolescent Psychiatry, Psychosomatics and Psychotherapy, Medical Faculty of the University of Cologne, Cologne, Germany. ${ }^{5}$ School of Child and Adolescent Cognitive Behaviour Therapy (AKiP), University Hospital of Cologne, Cologne, Germany. ${ }^{6}$ Department of Child and Adolescent Psychiatry and Psychotherapy, LWL-University Hospital Hamm, Ruhr-University Bochum, Hamm, Germany. ${ }^{7}$ Centre of Mental Health, Department of Child and Adolescent Psychiatry, Psychosomatics and Psychotherapy, University Hospital of Würzburg, Würzburg, Germany. ${ }^{8}$ Clinical Trials Unit Freiburg, Medical Centre - University of Freiburg, Faculty of Medicine, University of Freiburg, Freiburg, Germany. ${ }^{9}$ Department of Child and Adolescent Psychiatry and Psychotherapy, University Hospital Tübingen, Tübingen, Germany.

\section{Received: 14 August 2019 Accepted: 31 October 2019}

\section{Published online: 09 January 2020}

\section{References}

1. Banaschewski T, Becker K, Döpfner M, Holtmann M, Rosler M, Romanos M. Attention-deficit/hyperactivity disorder. A current overview. Dtsch Ärztebl Int. 2017;114(9):149-59.

2. Danckaerts M, Sonuga-Barke EJ, Banaschewski T, Buitelaar J, Döpfner M, Hollis C, et al. The quality of life of children with attention deficit/ hyperactivity disorder: a systematic review. Eur Child Adolesc Psychiatry. 2010;19(2):83-105.

3. Faraone SV, Asherson P, Banaschewski T, Biederman J, Buitelaar JK, RamosQuiroga JA, et al. Attention-deficit/hyperactivity disorder. Nat Rev Dis Primers. 2015;1:15020.

4. American Psychiatric Association. Diagnostic and statistical manual of mental disorders: DSM-5. Washington, DC: American Psychiatric Association; 2013.

5. World Health Organization. The ICD-10 classification of mental and behavioural disorders: Diagnostic criteria for research. Geneva: World Health Organization; 1993.

6. Huss M, Hölling H, Kurth BM, Schlack R. How often are German children and adolescents diagnosed with ADHD? Prevalence based on the judgment of health care professionals: results of the German health and examination survey (KiGGS). Eur Child Adolesc Psychiatry. 2008;17:52-8.

7. Lavigne JV, Lebailly SA, Hopkins J, Gouze KR, Binns HJ. The prevalence of ADHD, ODD, depression, and anxiety in a community sample of 4-year-olds. J Clin Child Adolesc Psychol. 2009;38(3):315-28.

8. Posner K, Melvin GA, Murray DW, Gugga SS, Fisher P, Skrobala A, et al. Clinical presentation of attention-deficit/hyperactivity disorder in preschool children: The preschoolers with attention-deficit/hyperactivity treatment study (PATS). J Child Adolesc Psychopharmacol. 2007;17(5):547-62.
9. Hanisch C, Freund-Braier I, Hautmann C, Janen N, Pluck J, Brix G, et al. Detecting effects of the indicated prevention programme for externalizing problem behaviour (PEP) on child symptoms, parenting, and parental quality of life in a randomized controlled trial. Behav Cogn Psychother. 2010;38(1):95-112.

10. Sonuga-Barke EJS, Koerting J, Smith E, MCCann DC, Thompson M. Early detection and intervention for attention-deficit/hyperactivity disorder. Expert Rev Neurother. 2011;11(4):557-63.

11. Sonuga-Barke EJ, Halperin JM. Developmental phenotypes and causal pathways in attention deficit/hyperactivity disorder: potential targets for early intervention? J Child Psychol Psychiatry. 2010;51(4):368-89.

12. Deutsche Gesellschaft für Kinder- und Jugendpsychiatrie und Psychotherapie (DGKJP). S3-Leitlinie zu ADHS im Kindes-, Jugend- und Erwachsenalter. URL: https://www.awmf.org/uploads/tx_szleitlinien/028-045 I_S3_ADHS_2018-06.pdf: AWMF online; 2017.

13. National Institute for Health and Care Excellence. Attention deficit hyperactivity disorder: diagnosis and management (NICE guideline). URL: https://www.nice.org.uk/guidance/ng87/resources/attention-deficithyperactivity-disorder-diagnosis-and-management-pdf-1837699732933; 2018.

14. Taylor E, Döpfner M, Sergeant J, Asherson P, Banaschewski T, Buitelaar J, et al. European clinical guidelines for hyperkinetic disorder - first upgrade. Eur Child Adolesc Psychiatry. 2004;13:7-30.

15. Greenhill L, Kollins S, Abikoff H, McCracken J, Riddle M, Swanson J, et al. Efficacy and safety of immediate-release methylphenidate treatment for preschoolers with ADHD. J Am Acad Child Adolesc Psychiatry. 2006;45(11): 1284-93.

16. Mulqueen JM, Bartley CA, Bloch MH. Meta-analysis: parental interventions for preschool ADHD. J Atten Disord. 2015;19(2):118-24.

17. Murray DW. Treatment of preschoolers with attention-deficit/hyperactivity disorder. Curr Psychiatry Rep. 2010;12(5):374-81.

18. Charach A, Carson P, Fox S, Ali MU, Beckett J, Lim CG. Interventions for preschool children at high risk for ADHD: a comparative effectiveness review. Pediatrics. 2013;131(5):e1584-604.

19. Comer JS, Chow C, Chan PT, Cooper-Vince C, Wilson LA. Psychosocial treatment efficacy for disruptive behavior problems in very young children: a meta-analytic examination. J Am Acad Child Adolesc Psychiatry. 2013; 52(1):26-36

20. Hautmann C, Hanisch C, Mayer I, Plück J, Döpfner M. Effectiveness of the prevention program for externalizing problem behaviour (PEP) in children with symptoms of attention-deficit/hyperactivity disorder and oppositional defiant disorder - generalization to the real world. J Neural Transm. 2008; 115(2):363-70

21. Plück J, Eichelberger I, Hautmann C, Hanisch C, Jaenen N, Döpfner M. Effectiveness of a teacher-based indicated prevention program for preschool children with externalizing problem behavior. Prev Sci. 2015;16(2): 233-41.

22. Hautmann C, Hoijtink H, Eichelberger I, Hanisch C, Pluck J, Walter D, et al. One-year follow-up of a parent management training for children with externalizing behaviour problems in the real world. Behav Cogn Psychother. 2009;37(4):379-96

23. Sonuga-Barke EJ, Brandeis D, Cortese S, Daley D, Ferrin M, Holtmann M, et al. Nonpharmacological interventions for ADHD: systematic review and meta-analyses of randomized controlled trials of dietary and psychological treatments. Am J Psychiatry. 2013;170(3):275-89.

24. Kazdin AE, Blase SL. Rebooting psychotherapy research and practice to reduce the burden of mental illness. Perspect Psychol Sci. 2011;6(1):21-37.

25. Klasen F, Meyrose AK, Otto C, Reiß F, Ravens-Sieberer U. Psychische Auffälligkeiten von Kindern und Jugendlichen in Deutschland. Monatsschr Kinderheilkd. 2017;165(5):402-7.

26. Franke N, Keown LJ, Sanders MR. An RCT of an online parenting program for parents of preschool-aged children with ADHD symptoms. J Atten Disord. 2016. https://doi.org/10.1177/1087054716667598.

27. Kierfeld F, Döpfner M. Bibliotherapy as a self-help program for parents of children with externalizing problem behavior. Z Kinder Jugendpsychiatr Psychother. 2006;34(5):377-85.

28. O'Brien M, Daley D. Self-help parenting interventions for childhood behaviour disorders: a review of the evidence. Child Care Health Dev. 2011. 37(5):623-37.

29. Tarver J, Daley D, Lockwood J, Sayal K. Are self-directed parenting interventions sufficient for externalising behaviour problems in childhood? 
A systematic review and meta-analysis. Eur Child Adolesc Psychiatry. 2014; 23(12):1123-37.

30. Kierfeld F, Ise E, Hanisch C, Görtz-Dorten A, Döpfner M. Effectiveness of telephone-assisted parent-administered behavioural family intervention for preschool children with externalizing problem behaviour: a randomized controlled trial. Eur Child Adolesc Psychiatry. 2013;22(9):553-65.

31. Ise E, Kierfeld F, Döpfner M. One-year follow-up of guided self-help for parents of preschool children with externalizing behavior. J Prim Prev. 2015; 36(1):33-40

32. Mokros L, Benien N, Mutsch A, Kinnen C, Schurmann S, Metternich-Kaizman TW, et al. Guided self-help interventions for parents of children with ADHD-concept, referral and effectiveness in a nationwide trial. An observational study. Z Kinder Jugendpsychiatr Psychother. 2015;43(4):275-86.

33. Dose C, Hautmann C, Buerger M, Schürmann S, Woitecki K, Döpfner M. Telephone-assisted self-help for parents of children with attention-deficit/ hyperactivity disorder who have residual functional impairment despite methylphenidate treatment: a randomized controlled trial. J Child Psychol Psychiatry. 2017;58(6):682-90.

34. Hautmann C, Dose C, Duda-Kirchhof K, Greimel L, Hellmich M, Imort S, et al. Behavioral versus nonbehavioral guided self-help for parents of children with externalizing disorders in a randomized controlled trial. Behav Ther 2018;49(6):951-65.

35. Döpfner M, Breuer D, Schurmann S, Metternich TW, Rademacher C, Lehmkuhl $\mathrm{G}$. Effectiveness of an adaptive multimodal treatment in children with attention-deficit hyperactivity disorder -- global outcome. Eur Child Adolesc Psychiatry. 2004;13(Suppl 1):1117-29.

36. Döpfner M, Ise E, Wolff Metternich-Kaizman T, Schurmann S, Rademacher C, Breuer D. Adaptive multimodal treatment for children with attention-deficit -/hyperactivity disorder: an 18 month follow-up. Child Psychiatry Hum Dev. 2015:46(1):44-56.

37. Döpfner M, Hautmann C, Dose C, Banaschewski T, Becker K, Brandeis D, et al. ESCAschool study: trial protocol of an adaptive treatment approach for school-age children with ADHD including two randomised trials. BMC Psychiatry. 2017;17(1):269.

38. Geissler J, Jans T, Banaschewski T, Becker K, Renner T, Brandeis D, et al. Individualised short-term therapy for adolescents impaired by attentiondeficit/hyperactivity disorder despite previous routine care treatment (ESCAadol)-Study protocol of a randomised controlled trial within the consortium ESCAlife. Trials. 2018;19:254.

39. Zinnow T, Banaschewski T, Fallgatter AJ, Jenkner C, Philipp-Wiegmann F, Philipsen A, et al. ESCAlate - Adaptive treatment approach for adolescents and adults with ADHD: study protocol for a randomized controlled trial. Trials. 2018;19:280.

40. Döpfner M, Plück J, Dose C, Eichelberger I, Schürmann S, Wolf M-KT. ADHSCoaching für Erzieherlnnen (3-6): Ein Selbsthilfeprogramm für Erzieherlnnen von Vorschulkindern mit ADHS-Problemen im Alter von 3 bis 6 Jahren. Köln: Klinik für Psychiatrie, Psychosomatik und Psychotherapie des Kindes- und Jugendalters an der Uniklinik Köln; 2015.

41. Döpfner M, Wolff Metternich-Kaizman T, Dose C, Katzmann J, Mokros L, Scholz K, et al. ADHS-Coaching für Eltern (3-6): Ein Selbsthilfeprogramm für Eltern von Vorschulkindern mit ADHS-Problemen im Alter von 3 bis 6 Jahren. Köln: Klinik für Psychiatrie, Psychosomatik und Psychotherapie des Kindes- und Jugendalters an der Uniklinik Köln; 2015.

42. Döpfner M, Schürmann S, Frölich J. Therapieprogramm für Kinder mit hyperkinetischem und oppositionellem Problemverhalten (THOP). 5th ed. Weinheim: Beltz; 2013.

43. Döpfner M, Schürmann S, Lehmkuhl G. Wackelpeter und Trotzkopf: Hilfen bei hyperkinetischem und oppositionellem Verhalten. Weinheim: Beltz; 2011.

44. Plück J, Wieczorrek E, Wolff Metternich T, Döpfner M. Präventionsprogramm für Expansives Problemverhalten (PEP): Ein Manual für Eltern und Erziehergruppen. Göttingen: Hogrefe; 2006.

45. Braun S. Entwicklung und Evaluation des Therapieprogramms zur Steigerung von Organisationsfähigkeit, Konzentration und Impulskontrolle bei Kindern mit ADHS. Universität Köln: THOKI-ADHS; 2018.

46. Döpfner M, Görtz-Dorten A. DISYPS-III : Diagnostik-System für Psychische Störungen nach ICD-10 und DSM-5 für Kinder und Jugendliche - III. Bern: Hogrefe; 2017

47. Guy W. ECDEU assessment manual for psychopharmology. USA: US Department of Health and Human Services; 1976.

48. Lange M, Kamtsiuris P, Lange C, Schaffrath Rosario A, Stolzenberg H, Lampert T. Messung soziodemographischer Merkmale im Kinder- und
Jugendgesundheitssurvey (KiGGS) und ihre Bedeutung am Beispiel der Einschatzung des allgemeinen Gesundheitszustands. Bundesgesundheitsblatt Gesundheitsforschung Gesundheitsschutz. 2007;50(5-6):578-89.

49. Petermann F, Wechsler D. Wechsler nonverbal scale of ability: [WNV]; Manual zur Durchführung und Auswertung; Übersetzung und Adaptation der WNV von David Wechsler. Frankfurt/M: Pearson; 2014.

50. Döpfner M, Plück J, Claudia K. Deutsche Schulalter-Formen der Child Behavior Checklist von Thomas M. Achenbach: CBCL 6-18R, TRF 6-18R, YSR 11-18R, Elternfragebogen über das Verhalten von Kindern u. Jugendlichen (CBCL/6-18R), Lehrerfragebogen über das Verhalten von Kindern u. Jugendlichen (TRF/6-18R), Fragebogen für Jugendliche (YRS/11-18R). Göttingen: Hogrefe; 2014.

51. Pasalich DS, Dadds MR, Hawes DJ, Brennan J. Do callous-unemotional traits moderate the relative importance of parental coercion versus warmth in child conduct problems? An observational study. J Child Psychol Psychiatry. 2011;52(12):1308-15

52. Weiss MD, Brooks BL, Iverson GL, Lee B, Dickson RA, Wasdell M. Reliability and validity of the Weiss Functional Impairment Rating Scale. In: Boston, 54th Annual Meeting of the American Academy of Child and Adolescent Psychiatry (abstract); 2007.

53. Ravens-Sieberer $\mathrm{U}$, Bullinger $\mathrm{M}$. Assessing health-related quality of life in chronically ill children with the German KINDL: first psychometric and content analytical results. Qual Life Res. 1998;7(5):399-407.

54. Strayhorn JM, Weidman CS. A Parent Practices Scale and its relation to parent and child mental health. J Am Acad Child Adolesc Psychiatry. 1988;27(5):613-8.

55. Imort S, Hautmann C, Greimel L, Katzmann J, Pinior J, Scholz K, et al. Der Fragebogen zum positiven und negativen Erziehungsverhalten (FPNE): Eine psychometrische Zwischenanalyse. Poster zum 32. In: Symposium der Fachgruppe Klinische Psychologie und Psychotherapie der DGPS, Braunschweig; 2014

56. Sanders MR, Markie-Dadds C, Tully LA, Bor W. The triple P-positive parenting program: a comparison of enhanced, standard, and self-directed behavioral family intervention for parents of children with early onset conduct problems. J Consult Clin Psychol. 2000;68(4):624-40.

57. Lovibond SH, Lovibond PF. Manual for the depression anxiety stress scales. 2nd ed. Sydney: Psychology Foundation of Australia; 1995.

58. Laucht M, Esser G, Baving L, Gerhold M, Hoesch I, Ihle W, et al. Behavioral sequelae of perinatal insults and early family adversity at 8 years of age. J Am Acad Child Adolesc Psychiatry. 2000;39(10):1229-37.

59. Goth K, Schmeck K. Das Junior Temperament und Charakter Inventar: JTCl; eine Inventarfamilie zur Erfassung der Persönlichkeit vom Kindergarten- bis zum Jugendalter nach Cloningers biopsychosozialem Persönlichkeitsmodell; Manual. Göttingen: Hogrefe; 2009.

60. Bölte S, Poustka F, Constantino JN. Assessing autistic traits: cross-cultural validation of the social responsiveness scale (SRS). Autism Res. 2008; (6):354-63.

61. Constantino J, Gruber C. The social responsiveness scale (SRS) manual. Los Angeles: Western Psychological Services; 2005.

62. Rösler M, Retz-Junginger $P$, Retz W, Stieglitz R-D. Homburger ADHS-Skalen für Erwachsene: HASE; Untersuchungsverfahren zur syndromalen und kategorialen Diagnostik der Aufmerksamkeitsdefizit-/Hyperaktivitätsstörung (ADHS) im Erwachsenenalter; Manual. Göttingen: Hogrefe; 2007.

63. Görtz-Dorten A, Döpfner M. Diagnose-Checklisten aus dem DiagnostikSystem für Psychische Störungen im Kindes- und Jugendalter (DISYPS-II) Gütekriterien und klinische Anwendung. Klin Diagn Eval. 2008;1:378-94.

64. Leucht $\mathrm{S}$, Fennema $H$, Engel R, Kaspers-Janssen M, Lepping P, Szegedi A. What does the HAMD mean? J Affect Disord. 2013;148(2-3):243-8.

65. Wilens TE, Prince JB, Waxmonsky J, Doyle R, Spencer T, Martelon M, et al. An open trial of sustained release bupropion for attention-deficit/hyperactivity disorder in adults with ADHD plus substance use disorders. J ADHD Relat Disord. 2010;1(3):25-35.

66. Canadian Attention Deficit Hyperactivity Disorder Resource Alliance. Canadian ADHD practice guidelines. 3rd ed. Toronto: Canadian Attention Deficit Hyperactivity Disorder Resource Alliance [CADDRA]; 2011.

67. Dose C, Hautmann C, Döpfner M. Functional impairment in children with externalizing behavior disorders: psychometric properties of the Weiss Functional Impairment Rating Scale-parent report in a German clinical sample. J Atten Disord. 2016; [Online first: Jul 28].

68. Gajria K, Kosinski M, Sikirica V, Huss M, Livote E, Reilly K, et al. Psychometric validation of the Weiss Functional Impairment Rating Scale-parent report form in children and adolescents with attention-deficit/hyperactivity disorder. Health Qual Life Outcomes. 2015;13:184. 
69. Plück J, Beiling M, Hautmann C, Kuschel A, Heinrichs N, Schmeck K, et al. Assessment of behavior problems in preschool children using the German version of CBCL/11/2-5. Diagnostica. 2013;59(3):155-66.

70. Achenbach TM, Resorla LA. Manual for the ASEBA preschool forms and profiles. Burlington: University of Vermont Department of Psychiatry; 2000.

71. KIDSCREEN Group Europe. The Kidscreen questionnaires: Quality of life questionnaires for children and adolescents: handbook. Lengerich: Pabst Science Publishers; 2006.

72. Harstick-Koll S, Kuschel A, Bertram H, Naumann S, Hahlweg C, Döpfner M. Erfassung der Lebensqualität von Vorschulkindern mit dem Kiddy-KINDL. Z Gesundheitspsychol. 2009;17:82-93.

73. Ravens-Sieberer U, Ellert U, Erhart M. Gesundheitsbezogene Lebensqualität von Kindern und Jugendlichen in Deutschland. Eine Normstichprobe für Deutschland aus dem Kinder- und Jugendgesundheitssurvey (KIGGS). Bundesgesundheitsblatt Gesundheitsforschung Gesundheitsschutz. 2007; 50(5-6):810-8.

74. Goodman R. The strengths and difficulties questionnaire: a research note. J Child Psychol Psychiatry. 1997;38(5):581-6.

75. Frick PJ, Hare RD. Antisocial process screening device: APSD. Toronto: MultiHealth Systems; 2001.

76. Bölte S, Poustka F. Skala zur Erfassung sozialer Reaktivität : SRS; dimensionale Autismus-Diagnostik; deutsche Fassung der Social Responsiveness Scale (SRS) von John N. Constantino und Christian P. Gruber. Bern: Huber; 2008.

77. Naumann S, Kuschel A, Bertram H, Heinrichs N, Hahlweg K. Förderung der Elternkompetenz durch Triple P-Elterntrainings. Prax Kinderpsychol Kinderpsychiatr. 2007;56(8):676-90.

78. Brown TA, Chorpita BF, Korotitsch W, Barlow DH. Psychometric properties of the Depression Anxiety Stress Scales (DASS) in clinical samples. Behav Res Ther. 1997;35(1):79-89.

79. Rösler M, Retz W, Retz-Junginger P, Thome J, Supprian T, Nissen T, et al. Instrumente zur Diagnostik der Aufmerksamkeitsdefizit

-/Hyperaktivitatsstorung (ADHS) im

ErwachsenenalterSelbstbeurteilungsskala (ADHS-SB) und Diagnosecheckliste (ADHS-DC). Nervenarzt. 2004;75(9):888-95.

80. Stringaris A, Goodman R, Ferdinando S, Razdan V, Muhrer E, Leibenluft E, et al. The Affective Reactivity Index: A concise irritability scale for clinical and research settings. J Child Psychol Psychiatry. 2012:53(11):1109-17.

81. Esser G. Mannheimer Elterninterview: MEl; strukturiertes Interview zur Erfassung von kinderpsychiatrischen Auffälligkeiten; Manual. Weinheim: Beltz-Test-GmbH; 1989

82. Rutter M, Quinton D. Psychiatric disorder-ecological factors and concepts of causation. In: McGurk M, editor. Ecological factors in human development. Amsterdam: Noord-Holland; 1997. p. 173-87.

83. Biederman J, Milberger S, Faraone SV, Kiely K, Guite J, Mick E, et al. Familyenvironment risk-factors for attention-deficit hyperactivity disorder - A test of Rutters indicators of adversity. Arch Gen Psychiatry. 1995;52(6):464-70.

84. Bryant FB, Smith BD. Refining the architecture of aggression: a measurement model for the Buss-Perry aggression questionnaire. J Res Pers. 2001;35(2):138-67.

85. Kiewitz C, Weaver JB. The aggression questionnaire. In: Reynolds RA, Woods R, Baker JD, editors. Handbook of research on electronic surveys and measurements. Hershey: Idea Group Publishing; 2007. p. 343-7.

86. Piacentini J, Woods DW, Scahill L, Wilhelm S, Peterson AL, Chang S, et al. Behavior therapy for children with tourette disorder a randomized controlled trial. JAMA. 2010;303(19):1929-37.

87. Döpfner M, Lehmkuhl G, Steinhausen H-C. KIDS1: Aufmerksamkeitsdefizitund Hyperaktivitätsstörung (ADHS). Göttingen: Hogrefe; 2006.

88. Romanos M, Weise D, Schliesser M, Schecklmann M, Löffler J, Warnke A, et al. Structural abnormality of the substantia nigra in children with attention-deficit hyperactivity disorder. J Psychiatry Neurosci. 2010;35(1):55-8.

89. Krauel K, Feldhaus HC, Simon A, Rehe C, Glaser M, Flechtner HH, et al. Increased echogenicity of the substantia nigra in children and adolescents with attention-deficit/hyperactivity disorder. Biol Psychiatry. 2010;68(4):352-8.

90. Berg D, Godau J, Walter U. Transcranial sonography in movement disorders. Lancet Neurol. 2008;7(11):1044-55.

\section{Publisher's Note}

Springer Nature remains neutral with regard to jurisdictional claims in published maps and institutional affiliations.

Ready to submit your research? Choose BMC and benefit from:

- fast, convenient online submission

- thorough peer review by experienced researchers in your field

- rapid publication on acceptance

- support for research data, including large and complex data types

- gold Open Access which fosters wider collaboration and increased citations

- maximum visibility for your research: over $100 \mathrm{M}$ website views per year

At BMC, research is always in progress.

Learn more biomedcentral.com/submissions 